\title{
Some General Properties of Stimulated Raman Propagation with Pump Depletion, Transiency and Dispersion
}

\author{
B. W. Shore \\ S. Lowder
}

M. A. Johnson

August 2, 1991

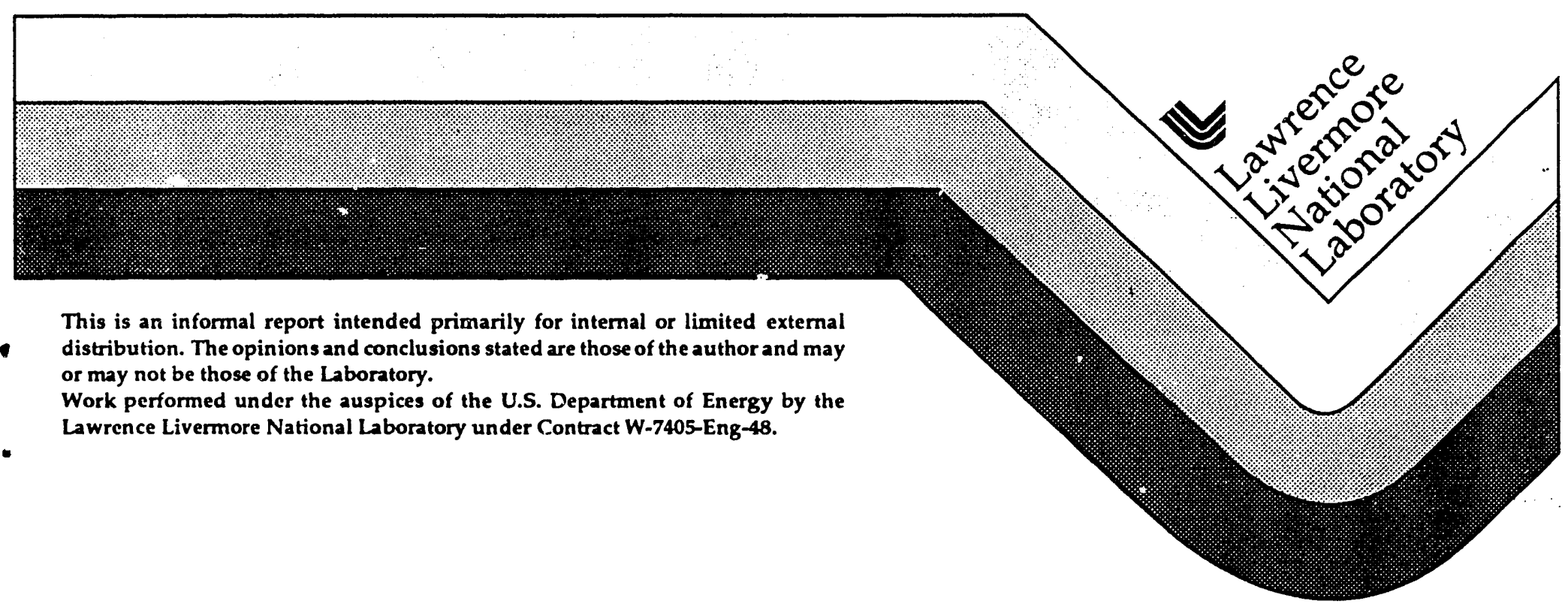




\section{DISCLAIMER}

This document was prepared as an acccount of work sponsored by an agency of the United States Government. Neither the United States Government nor the University of California nor any of their employees, makes any warranty, express or implied, or assumes any legal liability or responsibility for the accuracy, completeness, or usefulness of any information, apparatus, product, or process disclosed, or represents that its use would not infringe privately own rights. Reference herein to any specific commercial products, process, or service by trade name, trademark, manufacturer, or otherwise, does not necessarily constitute or imply its endorsement, recommendation, or favoring by the United States Government or the University of California. The views and opinions of authors expressed herein do not necessarily state or reflect those of the United States Government or the University of California, and shall not be used for advertising or product endorsement purposes.

This report has been reproduced directly from the best available copy.

Available to DOE and DOE contractors from the Office of Scientific and Technical Information

P.O. Box 62, Oak Ridge, TN 37831

Prices available from (615) 576-8401, FTS 626-8401

Available to the public from the National Technical Information Service

U.S. Department of Cummerce 5285 Port Royal Rd., Springfield, VA 22161 


\title{
Some General Properties of Stimulated Raman Propagation With Pump Depletion, Transiency and Dispersion
}

\author{
B. W. Shore, S. Lowder and M. A. Johnson \\ Lawrence Livermore National Laboratory \\ Livermore, CA 94550
}

This note considers some of the properties of the Stokes pulse that grows from a specified seed pulse in the presence of a strong pump pulse as it propagates through a dispersive atomic vapor.

1. We first present a generic dimensionless form for the coupled equations that govern the propagation of pump and Stokes fields for collinear plane-wave pulses. By treating the two fields we permit pump depletion. We include transient atomic response (as embodied in the Raman coherence), but neglect changes in atomic populations. (Thus our equations pertain to the regime in which atoms are more numerous than photons.)

The equations employ a gain length, a dispersion time $\tau_{\text {dis }}$, and a Raman coherence time (or memory time) $\tau_{R}$ as basic parameters: these two times, together with a singlephoton stationary-atom detuning $\Delta$, subsume the details of a particular atomic Raman transition and particular operating conditions. (The effects of Doppler shifts enters the equations through the coherence time.)

2. We discuss some general properties of these generic Raman propagation equations, and present illustrations of their solutions in the absence of dispersion. We comment on departures from exponential growth.

3. We then show examples of behavior when dispersion is present and the pump pulse has a bandwidth that exceeds the transform limit.

4. The illustrations presented here do not pertain to any specific atom (i.e. specific wavelengths and oscillator strengths) or to specific experimental conditions (i.e. number densities and pulse intensities.) To permit the connection between the present generic results and particular experiments we conclude by providing expressions for the gain length and dispersion time in terms of atomic number density, polarizabilities, oscillator strengths, statistical weights, transition frequencies, and polarization directions.

This work was pe: formed under the auspices of the United States Department of Energy by' the Lawrence Livermore National Laboratory under contract no. W-7405-Eng-48. 


\section{$\S 1$ Introduction and Definitions}

A strong pulse of radiation passing through an atomic vapor will generate pulses at a variety of other frequencies. We here consider those frequencies (Stokes radiation) that differ from the pump frequency by some two-photon (Raman) transition energy. For simplicity we consider only two frequencies, associated with the pump and a single Stokes pulse; we neglect anti-Stokes radiation as well as higher c:der Stokes radiation. In particular, we here consider the Stokes radiation as it grows from a weak seed pulse under the influence of a strong pump pulse, under idealized conditions of a dilute atomic vapor.

\section{$\S 1.1$ Basic Equations}

Consider the propagation of collinear pump and Stokes plane waves, both travelling along the $z$ axis, as described by optical carriers (at frequencies $\omega_{P}$ and $\omega_{S}$ ) and slowly varying electric-field envelopes $\mathscr{E}_{P}(z, t)$ and $\mathscr{E}_{S}(z, t)$, in the absence of population or field polarization changes. Let the time variable $t$ be measured relative to the front edge of a fiducial (light-speed) pulse, i.e. we employ a window that moves at the speed of light in vacuum, c. Under these conditions the equations governing the pump and Stokes fields may be written as

$$
\begin{aligned}
& \frac{\partial}{\partial z} \mathscr{E}_{S}(z, t)=-i \mathscr{K}_{S} \mathcal{E}_{S}(z, t) \\
& \quad-\pi \kappa_{S}(z) \int_{-\infty}^{\infty} d t^{\prime} \mathscr{G}\left(t-t^{\prime}\right) \mathcal{E}_{P}\left(z, t^{\prime}\right)^{*} \mathcal{E}_{S}\left(z, t^{\prime}\right) \mathcal{E}_{P}(z, t) \\
& \frac{\partial}{\partial z} \mathcal{E}_{P}(z, t)=-i \mathscr{K}_{P} \mathcal{E}_{P}(z, t) \\
& \quad+\pi \kappa_{P}(z) \int_{-\infty}^{\infty} d t^{\prime} \mathscr{G}\left(t-t^{\prime}\right) \mathcal{E}_{P}\left(z, t^{\prime}\right) \mathcal{E}_{S}\left(z, t^{\prime}\right)^{*} \mathcal{E}_{S}(z, t)
\end{aligned}
$$

where, as discussed below, $\mathscr{K}_{S}$ and $\mathscr{K}_{P}$ are linear operators responsible for dispersion, $\mathscr{G}(\tau)$ is the memory function for Raman coherence, $\pi$ is the Raman inversion $(\pi=-1$ for cold matter) and the coupling parameters $\kappa_{P}(z)$ and $\kappa_{S}(z)$ are proportional to frequencies, atomic number density $\cdot \hat{r}(z)$, and to the squares of polarizabilities. The envelopes are normalized so that pulse intensities are (cgs units)

$$
I_{S}(z, t)=\frac{c}{8 \pi}\left|\mathcal{E}_{S}(z, t)\right|^{2}, \quad I_{P}(z, t)=\frac{c}{8 \pi}\left|\mathcal{E}_{P}(z, t)\right|^{2} .
$$

The equations above present two contributions to puls: changes: a dispersive effect (involving $\mathscr{K}_{\lambda}$ ) and a Raman effect (involving coupling with another field). When the dispersive part does not include loss then the equations maintain a constant value of the quantity 


$$
\frac{I_{S}(z, t)}{\kappa_{S}(z)}+\frac{I_{P}(z, t)}{\kappa_{P}(z)}=\text { constant }
$$

This condition, iritepretable as conservation of photons, places an upper limit on the growth of a weak Stokes pulse.

\section{Objectives}

The equations above, taken with various simplifications, have been studied extensively, and various analytic solutions are known for special cases. Most commonly, the equations are solved without the dispersive terms, although special cases are soluble with dispersion. When the effect of the Stol is radiation upon the pump is negligible (the undepleted pump approximation) and the relaxation is homogeneous [i.e., the memory function $\mathscr{G}(\tau)$ is an exponential function of $\tau$, then there are known analytic solutions involving integrals of modified Bessel functions. When the relaxation time is very short, then simple solutions exist even with pump depletion (see below). Our concern here is with numerical solutions that include dispersion, transiency and pump depletion.

One desires solutions that emerge from a specified strong pump pulse and weak seed Stokes pulse at $z=0$. In particllar, one wishes to know how the pulse fluences

$$
F_{S}(z)=\int d t I_{S}(z, t), \quad F_{P}(z)=\int d t I_{P}(z, t)
$$

depend on various conditions, such as atomic density and temperature, pump pulse shape and fluence, and atomic polarizabilities and frequencies. These quantities, plotted as a function of distance $z$, reveal the growth of Stokes radiation and the loss of pump radiation. To understand details of the Raman process one requires, in addition, more detailed plots of pulse shape or pulse spectrum at a given distance.

Experiments with stimulated Raman scattering typically deal with two classes of initial conditions: either the Stokes seed is generated explicitly, and can be controlled along with the pump, or else the Stokes grows from spontaneous emission (quantum noise). Our concern in the present note will be the interplay between a few of the adjustable parameters that characterize pump and Stokes radiation. We shall not consider details of Stokes growth from noise (such considerations require, amongst other things, a specification of spatial geometry in order to count the number of spatial modes that should be incorporated into the idealized plane wave model). However, we shall consider examples of noisy Stokes seed pulses. 


\section{$\S 1.2$ Dispersive Effects}

The same induced dipole moments that produce Raman scattering, as parameterized by the quantities $\kappa_{\lambda}(z)$, also cause dispersive effects such as altered phase shifts, group velocity, and pulse distortion. Our interest is with a pump field that is nearly resonant with a singlephoton atomic resonance line, so that only a single excited state (or set of degenerate states) contributes to the atomic polarizability. Suppose the two-photon Raman transition links an initial state 1 with a final state 2 via off-resonant transition to an excited state 3 . The carriers for the pump transition $1 \longleftrightarrow 3$ and the Stokes transition $2 \longmapsto 3$ are each detuned from single-photon resonance by the angular frequency $\Delta$,

$$
\hbar \Delta=E_{3}-E_{1}-\hbar \omega_{P}=E_{3}-E_{2}-\hbar \omega_{S}
$$

so that (by definition) the two-photon Raman process is exactly resonant for the carriers. Under these conditions the effect of the dispersive operator $\mathscr{K}_{\lambda}$ may be written as

$$
\mathscr{K}_{\lambda} \mathcal{E}_{\lambda}(z, t)=K_{\lambda}(z)\left[1+\frac{i}{\Delta} \frac{\partial}{\partial t}+\ldots\right] \mathcal{E}_{\lambda}(z, t)
$$

where the ellipsis ... denotes an infinite series of time derivatives. For consistancy, the dispersion should be accompanied by absorption. This can be accomplished by making the replacement $\Delta \rightarrow \Delta-i \Gamma$ where $\Gamma$ is the single-photon loss rate. We here take this absorption to be negligible.

\section{Interpretation}

The $z$ dependence of $K_{\lambda}(z)$, like that of $\kappa_{\lambda}(z)$, enters as a proportionality to atomic density. For constant density $K_{\lambda}$ defines a length $1 / K_{\lambda}$ over which the envelope phase changes by one radian. The ratio $\omega_{\lambda} / K_{\lambda}$ is the conventional phase velocity. The first derivative term of this series expresses the incremental group velocity $v_{\lambda}^{\prime}$ with which a pulse shifts in the reference frame (moving with velocity $c$ ):

$$
\frac{1}{v_{\lambda}^{\prime}}=\frac{K_{\lambda}}{\Delta}
$$

Higher terms account for distortion of the pulse (e.g. amplitude modulation resulting from initial phase modulation). 


\section{$\S 1.3$ Alternative Variables}

As written. ine equations refer to a specific pair of transitions (whose oscillator strengths and frequencies enter through the parameters $\kappa_{P}$ and $\kappa_{S}$ ), to particular relaxation conditions (as embodied in the memory function), to particular density (through $\kappa_{S}$ and $\kappa_{P}$ ) and to a particular pump pulse (as embodied in pulse shape, pulse duration, and pulse fluence). It of ten proves advantageous to replace the variable $\mathscr{E}_{\lambda}$, whose dimensions are statvolts $/ \mathrm{cm}$ in the traditional cgs system, with alternative variables that permit generic discussion of results. For this purpose we introduce the amplitude variables

$$
Y_{S}(z, t)=a_{S} \mathscr{E}_{S}(z, t), \quad Y_{P}(z, t)=a_{P} \mathscr{E}_{P}(z, t)
$$

and coupling parameters

$$
g(z)=\frac{2 \kappa_{S}(z) \tau_{R}}{\left(a_{P}\right)^{2}}, \quad R=\frac{\kappa_{P}(z)}{\kappa_{S}(z)} \frac{\left(a_{P}\right)^{2}}{\left(a_{S}\right)^{2}}
$$

where $\tau_{R}$ is the Raman coherence time,

$$
\tau_{R}=\int_{-\infty}^{\infty} d \tau \mathscr{G}(\tau)
$$

sc that the fundamental equations $(1.1-1)$ become

$$
\begin{aligned}
& \frac{\partial}{\partial z} Y_{S}(z, t)=-i \frac{1}{2} \mathscr{K}_{S} Y_{S}(z, t)-w g(z) Y_{P}(z, t) \int_{-\infty}^{\infty} \frac{d t^{\prime}}{\tau_{R}} \mathscr{G}\left(t-t^{\prime}\right) Y_{P}\left(z, t^{\prime}\right)^{*} Y_{S}\left(z, t^{\prime}\right) \\
& \frac{\partial}{\partial z} Y_{P}(z, t)=-i \frac{1}{2} \mathscr{K}_{P} Y_{P}(z, t)+w R g(z) Y_{S}(z, t) \int_{-\infty}^{\infty} \frac{d t^{\prime}}{\tau_{R}} \mathscr{G}\left(t-t^{\prime}\right) Y_{P}\left(z, t^{\prime}\right) Y_{S}\left(z, t^{\prime}\right)^{*} .
\end{aligned}
$$

With these definitions the steady-state Stokes gain coefficient (an inverse length) for a (square) pulse of constant intensity passing through a uniform density is

$$
G_{s s}=2 \kappa_{S} \tau_{R}\left|\mathcal{E}_{P}(0, t)\right|^{2}=g\left|Y_{P}(0, t)\right|^{2}
$$

The intensity of a weak Stokes field, driven by such a square pulse, will grow exponentially by one neper $=\exp (1)$ in a distance $z=1 / G_{s s}$. 


\section{\$1.4 Particular Choice}

Both the magnitudes and dimensions of the new envelope variables $Y_{\lambda}$ remain to be chosen. It proves useful to express each envelope as a fraction of the mean initial pump envelope. Specifically, we let the variable $\left|Y_{\lambda}(z, t)\right|^{2}$ be the ratio of actual intensity to initial mean pump intensity (assuming ump pulse duration $T_{P}$ for fluence $F_{P}$ ),

$$
Y_{S}(z, t)=\mathcal{E}_{S}(z, t) \sqrt{\frac{c T_{P}}{8 \pi F_{P}(0)}}, \quad Y_{P}(z, t)=\mathcal{E}_{P}(z, t) \sqrt{\frac{c T_{P}}{8 \pi F_{P}(0)}}
$$

With these definitions we have the assignments

$$
\left(a_{S}\right)^{2}=\left(a_{P}\right)^{2}=\frac{c T_{P}}{8 \pi F_{P}(0)}
$$

and the dimensionless envelope variables $Y_{\lambda}$ are normalized such that at $z=0$

$$
\int d t\left|Y_{S}(0, t)\right|^{2}=T_{P} \frac{F_{S}(0)}{F_{P}(0)}, \quad \int d t\left|Y_{P}(0, t)\right|^{2}=T_{P}
$$

With this normalization a (square) pump pulse of constant amplitude and duration $T_{P}$ is characterized by an envelope of unit amplitude

$$
Y_{P}(0, t)=1 \quad \text { for } 0<t<T_{P} .
$$

The pulse fluences (time integrated intensities) at position $z$ are

$$
F_{S}(z)=\frac{F_{P}(0)}{T_{P}} \int d t\left|Y_{S}(z, t)\right|^{2}, \quad F_{P}(z)=\frac{F_{P}(0)}{T_{P}} \int d t\left|Y_{P}(z, t)\right|^{2}
$$

and the propagation parameters are

$$
g(z)=2 \kappa_{S}(z) \frac{8 \pi F_{P}(0)}{c T_{P}} \tau_{R}, \quad R=\frac{\omega_{P}}{\omega_{S}} .
$$

When the atomic number density is uniform then $g$ is the steady-state gain coefficient for a pulse of constant intensity and duration $T_{P}$ :

$$
g=G_{s s} \quad \text { (square pulse, uniform density) }
$$

Either the dispersion length $1 / K_{\lambda}$ or the Stokes steady-state gain length $1 / g$ may be taken as the unit of length. Because our primary concern is with the growth of Stokes radiation, we choose to measure distance in units of $1 / \mathrm{g}$ by introducing a (dimensionless) distance scale (sometimes termed the gain-length product) 


$$
Z=\int_{0}^{z} d z g\left(z^{\prime}\right)=\int_{0}^{z} d z \kappa_{S}\left(z^{\prime}\right) \frac{16 \pi F_{P}(0)}{c T_{P}} \tau_{R}
$$

The integration reduces to the product $g z$ when the atomic density remains constant, and it permits the treatment of more general situations in which density varies along the path. pulse,

In place of the dispersive length scale $K_{\lambda}$ we introduce a dispersion time for the pump

$$
\tau_{d i s}=\frac{1}{g v_{P}^{\prime}}=\frac{K_{P}(z)}{g(z) \Delta}=\frac{K_{P}(z)}{\kappa_{S}(z)} \frac{c T_{P}}{16 \pi F_{P}(0)} \frac{1}{\tau_{R} \Delta}
$$

and a ratio of Stokes to pump dispersion,

$$
\zeta=\frac{K_{S}(z)}{K_{P}(z)}
$$

\section{Interpretation}

The value $Z=1$ corresponds to a distance (the steady-state gain length) such that for short Raman relaxation time in a uniform medium a square pump pulse, of fluence $F_{P}(0)$ and duration $T_{P}$, will produce one neper of growth in a weak Stokes pulse.

When the pump pulse has propagated this distance $(z=1 / g$, one steady-state Stokes gain length, $Z=1$ ) the incremental group velocity will cause the pulse to appear to drift by time $\tau_{d i s}$ in the (moving) roference frame. In the same distance the Stokes pulse will tend to drift by $\zeta \tau_{\text {dis }}$.

\section{Basic Equations}

With these definitions the fundamental equations read

$$
\begin{aligned}
\frac{\partial}{\partial Z} Y_{S}(Z, t)= & i \zeta\left[\tau_{d i s} \Delta\right]\left[1+\frac{i}{\Delta} \frac{\partial}{\partial t}+\ldots\right] Y_{S}(Z, t) \\
& -\frac{1}{2} \pi Y_{P}(Z, t) \int_{-\infty}^{\infty} \frac{d t^{\prime}}{\tau_{R}} \mathscr{Y}\left(t-t^{\prime}\right) Y_{P}\left(Z, t^{\prime}\right)^{*} Y_{S}\left(Z, t^{\prime}\right) \\
\frac{\partial}{\partial Z} Y_{P}(Z, t)= & i\left[\tau_{d i s} \Delta\right]\left[1+\frac{i}{\Delta} \frac{\partial}{\partial t}+\ldots\right] Y_{P}(Z, t) \\
& +\frac{1}{2} \Vdash R Y_{S}(Z, t) \int_{-\infty}^{\infty} \frac{d t^{\prime}}{\tau_{R}} \mathscr{G}\left(t-t^{\prime}\right) Y_{P}\left(Z, t^{\prime}\right) Y_{S}\left(Z, t^{\prime}\right)^{*}
\end{aligned}
$$


These dimensionless equations are the ones we consider. They involve dimensionless pulse shapes $Y_{\lambda}(z, t)$ whose time-averaged magnitude is fixed by Eqn. (1.4-3). Full specification of the incident pulses requires a pulse duration $T_{P}$ (say the interval between half power points of the intensity), a bandwidth and other such parameters. All atcmic properties enter through the dimensionless memory function $\mathscr{G}(\tau)$, the ratios $R=\left(\omega_{S}\right) /\left(\omega_{p}\right)$ and $\zeta$, and the time scales $\tau_{R}, \tau_{d i s}$ and $1 / \Delta$. Because the Stokes dispersion comes primarily from excitation of level 2 (whereas the pump dispersion comes from initially populated level 1 ), propagation through cold matter has $\zeta=0$. The mean population difference between levels 1 and 2 enters as the fixed Raman inv rsion $w$.

\section{Conservation of Photons} quantity

It is noteworthy that these equations maintain a fixed value of the dimensionless

$$
G(t)=\left|Y_{P}(Z, t)\right|^{2}+R\left|Y_{S}(Z, t)\right|^{2}
$$

When the incident Stokes field is much weaker than the pump field (as is the case of interest) we have the properties

$$
\begin{aligned}
& G(t) \simeq\left|Y_{P}(0, t)\right|^{2} \simeq R\left|Y_{S}(\infty, t)\right|^{2} \\
& \int d t G(t)=T_{P} .
\end{aligned}
$$

The conserved photon flux (photons per unit area per unit time) is

$$
\Phi(t)=\frac{I_{P}(0, t)}{\hbar \omega_{P}}=\frac{F_{P}(0)}{\hbar \omega_{P} T_{P}} G(t)
$$

The total number of photons per unit area (also conserved) is

$$
\int d t \Phi(t)=\frac{F_{P}(0)}{\hbar \omega_{P}} .
$$

These conservation properties provide a useful check on numerical results.

Simplification: $R=1$

Without altering significant physics we may choose $R=1$. This is equivalent to choosing the Stokes variable to be

$$
Y_{S}(z, t)=\sqrt{R} Y_{S}(z, t)
$$

and amounts to using photon numbers rather than intensities as the variables of direct interest. 


\section{$\S 1.5$ Dispersionless Steady State}

An important limiting case of Raman propagation occurs when dispersion is negligible and the relaxation time is much shorter than other time scales. Under these conditions, and for cold matter $(w=-1)$ we deal with the steady-state dispersionless equations

$$
\begin{aligned}
& \frac{\partial}{\partial Z} Y_{S}(Z, t)=+\frac{1}{2}\left|Y_{P}(Z, t)\right|^{2} Y_{S}(Z, t) \\
& \frac{\partial}{\partial Z} Y_{P}(Z, t)=-\frac{1}{2} R\left|Y_{S}(Z, t)\right|^{2} Y_{P}(Z, t) .
\end{aligned}
$$

These coupled equations have the well-known analytic solution

$$
\left|Y_{S}(Z, t)\right|^{2}=\left|Y_{S}(0, t)\right|^{2} \frac{\left[\left|Y_{P}(0, t)\right|^{2}+R\left|Y_{S}(0, t)\right|^{2}\right] \exp [G(t) Z]}{\left|Y_{P}(0, t)\right|^{2}+R\left|Y_{S}(0, t)\right|^{2} \exp [G(t) Z]}
$$

where

$$
G(t)=\left|Y_{P}(0, t)\right|^{2}+R\left|Y_{S}(0, t)\right|^{2}
$$

is proportional to the incident photon flux. This Stokes field exhibits exponential growth at small distances $(G(t) g z \ll 1)$,

$$
\left|Y_{S}(Z, t)\right|^{2} \rightarrow\left|Y_{S}(0, t)\right|^{2} \exp [G(t) Z]
$$

and saturation at large distances $(G(t) g z \gg>1)$,

$$
\left|Y_{S}(Z, t)\right|^{2} \rightarrow\left|Y_{P}(0, t)\right|^{2}+R\left|Y_{S}(0, t)\right|^{2}
$$

This analytic solution provides a convenient reference for examination of propagation under less restricted idealization. 


\section{$\S 2$ Numerical Examples, No Dispersion}

We next present a numbor of examples of solutions to these propagation equations, obtained by numerical integration, for a variety of conditions. These examples to not necessarily represent any particular Raman transitions in particular atoms under any proposed experimental conditions. Rather, they are chosen to illustrate general principles.

In all of the computations presented here the memory function $\mathscr{G}(\tau)$ is taken to be the exponential $\exp \left[-\tau / r_{R}\right]$, corresponding to a Lorentzian profile for coherence relaxation. The Lorentz profile is appropriate for homogeneous relaxation. We have examined other choices, including the conventional Doppler profile that leads to a Gaussian form for the memory function. Although there are observable differences between different forms for the memory function, the qualitative features that we discuss here occur for any form.

For simplicity we take the ratio of Stokes to pump frequencies to be unity for all calculations, and we always assume that the Stokes wave propagates without dispersion,

$$
R=1, \quad \zeta=0 .
$$

We take as our unit of time the nanosecond (rather than, say, the pulse duration or the relaxation time). We take the pulse duration $T_{P}$ to be the interval between the half power points of the intensity $I_{P}(0, t)$ of the incident pulse.

To exhibit pump depletion phenomena we take the initial Stokes seed fluence to be smaller than the initial pump fluence by $\exp (-10)=4.5 \times 10^{-5}$, so that for a steady-state square pulse and no pump depletion the initial seed would become equal to the pump after 10 gain lengths. We typically show results of propagation to $Z=20$, twice this saturation length.

We present plots of several things. To exhibit the exponential growth of Stokes fluence we show, as a function of gain length $Z$, the relative fluences of pump and Stokes pulses, expressed as photon numbers relative to the incident pump fluence. We express these fluences as relative photon numbers (in these units the incident pump fluence is 1.0 and the incident Stokes fluence is $e^{-10}$ ) On separate plots we show the square of the relative pulse magnitudes, $\left|Y_{\lambda}(Z, t)\right|^{2}$, together with the relative power spectra (the absolute square of the Fourier transform of the pulse envelope). For a few cases we show the envelope magnitude and phase, and the magnitude of the Fourier transform of the pulse envelope. Because we express time in nanoseconds, the frequency scale is Ghz.

\section{Dispersionless Examples}

For the calculations of this section we neglect all pump dispersion, $\tau_{\text {disp }}=0$, and we exhibit pulses in a window $200 \mathrm{~ns}$ long. Because there is no dispersion, pulses remain centered in this window. We typically take the pump pulse to have duration $T_{P}=100 \mathrm{~ns}$. 


\section{§2.1 Saturation Effects}

We first consider propagation with a short relaxation time. We construci a pump pulse with constant phase of duration $T_{P}=100 \mathrm{~ns}$, so that the pulse bandwidth is transform limited. We take the Raman coherence time (the memory time) to be $\tau_{R}=1 \mathrm{~ns}$, so that the relaxation time is much shorter than the pulse duration but is not zero. We expect that, under these circumstances, the pulses will be governed to a good approximation by the steadystate equations. In particular, we expect to see initially exponential growth, followed by saturation.

We consider a pulse whose envelope is a gauss- 16 hypergaussian [i.e. whose amplitude varies with time as the exponential of $\left.\left(t-t_{0}\right)^{16}\right]$. This is nearly, but not exactly, a square pulse. We take a Stokes seed whose shape and phase is identical with the pump. Then we find that the Stokes fluence, like the Stoikes intensity, obeys very closely the saturation solution. The pump fluence remains fairly constant at first, but ultimately converts, within a few gain lengths, to Stokes radiation. For the particular choice of seed used here, an initial fluence of $e^{-10}$, the conversion is half completed after 10 gain lengths.

see Figure $1 a$

This figure displays four curves. First, it shows (dashed and labelled "pump") the pump fluence as it starts from given incident value (a relative photon number 1.0 ) and subsequently converts to Stokes radiation. Next, it shows (solid and unlabeled) the numerically calculated Stokes fluence (starting from $e^{-10}$ ). The dashed curve (long dashes) labelled "steady" is the Stokes fluence that would have occurred if the coherence time had been infinitisimally short. The dashed curve (short dashes) labelled "square" is the analytic expression for the Stokes fluence that would occur if the coherence time had been infinitisimal and the pump had been exactly square (with the given fluence).

It can be seen from this figure that for this particular example the assumption of steady-state growth gives an excellent description of the Stokes fluence, and that the assumption of a square pulse introduces only a small (but quite perceptible) error.

The exponential nature of the initial Stokes growth can be seen much more clearly on a semilog plot of Stokes fluence vs. distance. On such a plot we can also see clearly that, whereas the steady-state solution predicts complete conversion of pump to Stokes radiation, the actual pump depletion is not complete. The discrepancy is quite obvious on a logarithmic plot, but is not so noticeable on a linear plot.

see Figure $1 b$

The reason for lack of complete pump depletion can be seen in a picture of the pump envelope as it appears at large Z. Because the memory time is not infinitismal, there is an interval at the beginning and the end of the pulse, a few memory times in duration, during which the pump is not completely depleted. These remnants deplete very slowly with distance.

see Figure $1 c$ 


\section{§2.2 Pulse Shape Effects}

Next we consider cases, still with short memory time and without dispersion, in which the initial pump pulse does not maintain constant amplitude. As before, we take the Stokes seed and initial pump pulse to have identical initial envelopes, and to have constant phase. In this case we observe very appreciable differences between the actual numerical solution and the analytic solution for the square pulse.

see figure $2 a$

We find that the Stokes initially grows exponentially, but the gain length is longer than one would estimate for a square pulse of equal fluence. Saturation occurs, but at a later time than for the equivalent square pulse.

see Figure $2 b$

Evidently the gain length, defined in terms of a square pulse, does not accurately describe the fluence of a pulse that is not square. This is to be expected: the time integral of an exponentiated time variation is not the same as the exponential of a time integral.

Plots of pulse intensity for pump envelopes that are not square show the effects of transiency on Stokes shape and on pump depletion.

see figure $2 c$ 


\section{$\S 2.3$ Correlation Between Seed and Pump}

When the initial Stokes seed differs in phase or in shape from the initial pump envelope then the initial growth of the fluence is not exponential: only after a few gain lengths does exponential growth of fluer.e set in.

As an example, we eximine, for a hypergaussian pump pulse with constant phase, a Stokes seed that is constant (and has constant phase) within the entire time window. Only that portion of the Stokes envelope that overlaps the pulse can undergo growth; this portion grows exponentially. Once appreciable growth occurs, the Stokes puise does not depend on the duration of the seed.

see Figure 3

As another example of the importance of correlation between pump and Stokes seed, we modify the previous case by introducing a varying phase for the pump, so that the pump bandwidth exceeds the transform limit. We expect that only a fraction of the pump spectrum will overlap the Stokes seed and produce exponential growth.

see Figure $4 a$

The presence of pump phase variation, together with a finite memory time, produces a modulation of the amplitude of the Stokes pulse. The period of this modulation coincides with the warble period of the pump. This amplitude variation, in turn, causes a corresponding modulation of the pump depletion.

see Figure $4 b$

In each of these cases the behavior is (approximately) as if only some fraction of the Stokes seed fluence undergoes exponential growth. An estimate of this fraction is the Stokes-pump correlation,

$$
|c|^{2}=\frac{\left|\int d t \mathcal{E}_{P}(t)^{*} \mathscr{E}_{S}(t)\right|^{2}}{\int d t\left|\mathcal{E}_{P}(t)\right|^{2} \times \int d t\left|\mathcal{E}_{S}(t)\right|^{2}}
$$

This number has been used to draw the fluence curves for square-puise propagation in the previous two figures: Those curves are drawn for a hypothetical square pulse whose tluence is smaller than the actual fluence by the amount $|c|^{2}$.

Although the correlation is useful in providing a bound, 11 toes not always give a close estimate. When the seed pulse has large amplitude fluctuations the final pulse will also exhibit amplitude fluctuations. As extreme example, we seed this same pump pulse 
with a realization of a completely random seed, i.e. an envelope whose succession of complex values are Gaussian random variables with zero mean. Such a seed is what one would use to model growth from spontaneous emission, although in that case the seed would typically be smaller than the pump by $\exp (-30)$ rather than the present $\exp (-10)$. Again we observe an initially slow growth of fluence, followed by exponential growth and saturation. The effective portion of the seed is, in this case, quite small, and exponential growth does not start until the pulses have propagated some 5 gain lengths.

\section{see Figure $5 a$}

The behavior of a random seed exhibits interesting details that are not evident on plots of fluence. Some of the irregularity of the initial Stokes pulse is maintained as it grows. This, in turn, leads to irregularity in pump depletion. Different samples of random seeds will exhibit different details of time dependence, although these details tend to be lost in logarithmic plots of fluence.

see Figure $5 b$ 


\section{$\S 2.4$ Stokes Phase}

As long as the memory time is shorter than phase or amplitude variations of the pump envelnne (and there is is dispersion), then the Stokes envelope does not depend on pump phise. This we can see from the steady-state equations. Whatever initial phase is present in the Stokes seed will be preserved with growth. However, a nonzero memory time will transfer to the Stokes envelope the phase of the pump envelope. With sufficient distance, the Stokes pulse will acquire the pump phase.

Such behavior can be seen in the Stokes field of the previous example. The initial random Stokes phase becomes replaced by the pump phase (in this case a constant phase).

see Figure 6

As another example, consider the previous cases (100ns hypergaussian pump pulse, Ins relaxation time) with a Stokes seed that is constant in amplitude and phase. We impose on the pump a sinusoidal phase variation (a warble), such that the bandwidth of the pump becomes an appreciable fraction of the available frequency window. The propagating Stokes becomes clearly "marked" with this pump phase.

see Figures $7 a$ (pump) and $7 b$ (stokes)

The presence of pump modulation that does not match the Stokes phase, like the presence of Stokes seed fluctuations that do not match the pump, slows the initial growth of the Stokes pulse, as noted previously. (When pump and Stokes share the same modulation, then the Stokes growth is the same as with narrow bandwith pulses.)

Because the memory time is not infinitisimally short in these examples, some phase transfers from pump to Stokes. This results in an amplitude modulation of the growing Stokes pulse and, when pump depletion sets in, an amplitude modulation of the residual pump pulse, as noted in a previous figure. 


\section{$\S 2.5$ Transiency}

When the memory time becomes an appreciable fraction of the pulse duration, then steady-state equations become a poor guide to the behavior of propagation. The leading edge of the pump pulse is not as effective in producing Stokes radiation as is the subsequent portion of the pulse: the induced dipole moment responsible for Stokes radiation is zero when the pump first arrives, and only after a few memory times does it stabilize at the value prescribed by the pump. Such transiency diminishes the growth of pulse fluence; only a portion of the pump produces exponential growth. The effect is not simply a decrease in the rate of exponential growth, as it is with pulse shapes that are not square. Rather the fluence growth is slower than any exponential. We illustrate this effect with examples of a hypergaussian pump, zero phase, and constant Stokes seed, for coherence times $\tau_{R}=10 \mathrm{~ns}$ (10 percent of the pulse duration) and $\tau_{R}=50 \mathrm{~ns}$ (half the pulse duration).

With the shorter of these times, $\tau_{R}=10 \mathrm{~ns}$, the departure from steady-state exponential growth is quite noticeable in a semilog plot of Stokes fluence.

see Figure $8 a$

Plots of pulse shape reveal, as would be expected, slower rise of the Stokes pulse and consequently a larger remnant of the leading edge of the pump pulse. The plots also reveal, at the leading edge of the Stokes pulse, a tendency toward a ripple.

see Figure $8 b$

The effects of transiency become quite pronounced as the coherence time becomes comparable to the pulse duration, as in the examples for which $r_{R}=50 \mathrm{~ns}$. The Stokes fluence growth is much slower than with shorter coherence times.

see Figure $9 a$

For long memory times the Stokes pulse acquires a characteristic transient shape, so long as pump depletion remains negligible.

see Figure $9 b$

As pump depletion sets in, the Stokes and pump each exhibit the ripple behavior noted above.

see Figure $9 c$ 


\section{$\S 3$ Dispersion}

This section presents examples of Raman processes in the presence of dispersion. We now must consider the dispersion time $\tau_{d i s}$ and the pump bandwidth as well as the memory time $\tau_{R}$ and the pulse duration $T_{P}$.

As above, we take $R=1,5=0$. For illustrative purposes we take the detuning $\Delta=50$ $n s^{-1}$ (corresponding to $2 \pi \Delta=8 \mathrm{Ghz}$ ).

Because dispersion causes the pump pulse to drift in the reference window, it is necessary to extend the window further than for nondispersive calculations. We here take a time window of $300 \mathrm{~ns}$. In the distance $Z=20$ a dispersion time of $\tau_{\text {dis }}=1 \mathrm{~ns}$ will produce, by differential group velocity, a drift of $20 \mathrm{~ns}$.

\section{\$3.1 Dispersion Without Modulation}

Dispersion alone has the following effects. First, it adds a constant to the preexisting phase. This has no observable consequence for Raman processes. Second, it causes a drift of the entire envelope relative to the temporal location it would have in vacuum. This is the effect of group velocity. Third, it introduces distortion (modulation) of the preexisting phase and amplitude. This occurs particularly when the pulse has phase variation (i.e. appreciable bandwidth).

Dispersive propagation of an unmodulated pulse delays the pulse, in the reference window, but does not distort the envelope. The pulse delay actually permits more effective growth of the Stokes radiation at the back end of the pump. Consequently the fluence growth does not differ noticeably from that of nondispersive propagation. As an example, consider the pump to be a hypergaussian (nearly square) with constant phase and a dispersion time of $\tau_{\text {disp }}=5 \mathrm{~ns}$. The resulting fluence growth of Stokes radiation is similar to steady-state exponential growth, but there are apparent differences as well. In particular, there is little pump depletion.

see Figure 10a

The behavior of these pulses can be understood from plots of pulse shape. In the absence of Stokes radiation, dispersion moves the pump pulse steadily in the reference window, at a speed of 5ns per gain length, without noticeable change of shape. The growing Stokes pulse depletes this pulse slightly at first.

see Figure 10b

As propagation progresses the Stokes removes energy from the front edge of the pump pulse but is unable to remove energy from the back edge. Consequently the tail of the pump, which moves steadily in the reference window, remains less depleted.

see Figure 10c

Eventually the pump pulse moves entirely out of the original window, and is not able to produce further Stokes growth.

see Figure 10d. 


\section{§3.2 Dispersion with modulation}

We next consider dispersive propagation of a nump whose bandwidth is appreciable, specifically a pulse whose phase varies sinusoidally. Dispersion causes the pump to develop amplitude modulation. The resulting growth of Stokes fluence is noticably slower than exponential.

\section{see Figure $11 a$}

An examination of pulse envelopes reveals the importance of phase modulation. Within a few gain lengths the sinuscidal phase modulation becomes a sinusoidal amplitude modulation. This pump amplitude variation leads, in turn, to strong modulation of the Stokes amplitude.

\section{see Figure $11 b$}

As propagation continues, and the pump modulation becomes more severe (as well as drifting in the reference window), the pump becomes less effective at producing Stokes radiation.

see Figure $11 c$ 


\section{$\S 3.3$ Summary}

When the relaxation time is short and dispersion is negligible, the simple saturable analytic expressions give reliable estimates of Stokes fluence growth. The Stokes fluence grows exponentially, after an initial period of slow growth, until it bcomes comparable to the initial pump fluence.

Departures cf pulse shape from square can be accounted for by introducing an aliernative estimate of pulse duration, in place of the half-power points.

When the Stokes seed differs from the pump pulse, the correlation function provides a rough estimate of the fraction of the seed that will undergo growth.

The Stokes pulse tends to acquire the shape and the phase of the pump.

Amplitude noise on the Stokes seed remains in the amplified pulse.

Both transiency and dispersion (for pump with appreciable bandwidth) cause the Stokes fluence growth to be slower than exponential. 


\section{§4 Raman Parameters}

To establish connection between the generic results of the dimensionless equations and specific experiments we need to evaluate the gain length and the dispersion time. These two quantities, through their definition in terms of the quantities $\mathscr{K}_{\lambda}$ and $\kappa_{\lambda}(z)$ that appear in the original equations, serve as the principle collection of depencence upon density, wavelength and oscillator strengths.

\section{$\S 4.1$ Memory Function}

We also require the memory function $\mathscr{G}(\tau)$. By definition, this function is the Fourier transform of the two-photon Doppler distribution $g_{12}(\delta)$ :

$$
\mathscr{G}(\tau)=\int d \delta \exp \left[-i \tau \delta-\Gamma_{12} \tau\right] g_{12}(\delta)
$$

The homogeneous relaxation rate $\Gamma_{12}$ that occurs here combines the collisional relaxation of the Raman coherence with a radiative decay rate from level 2. Both of these are much less than the Doppler width for tenuous vapor and opt $\sim$ - $>$ excitation, but they are the dominant processes at higher density (as is assumed for the plots of the present paper). The normalizations are

$$
\int d \delta g_{12}(\delta)=1=\mathscr{G}(0)
$$

so that the time integral of the memory function is the Raman coherence time:

$$
\tau_{R}=\int d \tau \mathscr{G}(\tau)=\pi_{\mathscr{I}_{12}}(0)
$$

For a conventional (Gaussian) Doppler profile whose full width at half maximum is $\Delta \nu_{D}$ hertz the coherence time is

$$
\tau_{R}=\sqrt{\frac{\ln 2}{\pi}} \frac{1}{\Delta \nu_{D}}
$$

The profile $g_{12}(\delta)$ need not be Gaussian; it may indeed be Lorentzian or hypergaussian. Whatever the functional form of $g_{12}(\delta)$ and $\mathscr{G}(\tau)$, Eqn. $(4.1-3)$ defines the memory time. 


\section{$\S 4.2$ Propagation Parameters: Polarizabilities}

The dispersive parameters $K_{\lambda}(z)$ and the Raman gain parameters $\kappa_{\lambda}(z)$ may be written in terms of averaged single-atom polarizabilities $\alpha_{p p^{\prime}}\left(\lambda, \lambda^{\prime}\right)$ and atomic number density $\mathcal{N}$ $=\mathcal{N}_{1}+\mathcal{N}_{2}$

$$
\begin{aligned}
& K_{P}(z)=\frac{2 \pi \omega_{P}}{c} \mathcal{N}_{1}(z)\left\{\alpha_{11}(P, P)\right\} \\
& K_{S}(z)=\frac{2 \pi \omega_{S}}{c} \mathcal{N}_{2}(z)\left\{\alpha_{22}(S, S)\right\} \\
& \kappa_{S}(z)=\frac{\pi \omega_{S}}{2 \hbar c} \mathcal{N}(z)\left\{\left|\alpha_{12}(P, S)\right|^{2}\right\} \\
& \kappa_{P}(z)=\frac{\omega_{P}}{\omega_{S}} \kappa_{S}(z)
\end{aligned}
$$

From these formulas we evaluate the gain coefficient and dispersion time, defined as

$$
\begin{aligned}
& g(z)=2 \kappa_{S}(z) \frac{8 \pi F_{P}(0)}{c T_{P}} \tau_{R} \\
& \tau_{d i s}=\frac{K_{P}(z)}{\kappa_{S}(z)} \frac{c T_{P}}{8 \pi F_{P}(0)} \frac{1}{\tau_{R} \Delta}
\end{aligned}
$$

where $F_{P}(0)$ is the incident pump fluence and $T_{P}$ is the initial pump pulse width. The results are the formulas

$$
\begin{aligned}
& g(z)=\left[\frac{8 \pi^{2}}{\hbar c^{2}}\right] \mathcal{N}(z)\left\{\left|\alpha_{12}(P, S)\right|^{2}\right\} \frac{F_{P}(0)}{T_{P}} \omega_{S} \tau_{R} \\
& \tau_{\text {dis }}=\left[\frac{\hbar c}{2 \pi}\right] \frac{T_{P}}{F_{P}(0)} \frac{\left\{\alpha_{11}(P, P)\right\}}{\left\{\left|\alpha_{12}(P, S)\right|^{2}\right\}} \frac{\mathcal{N}_{1}}{\mathcal{N}} \frac{\omega_{P}}{\omega_{S}} \frac{1}{\tau_{R} \Delta}
\end{aligned}
$$




\section{$\S 4.3$ Dipole Transition Strengths}

For near resonant propagation involving a single virtual level (level 3) and in whic'. the detuning $\Delta$ is constrained by Eqn. $(1.2-1)$, the polarizabilities are expressible in "orms of the Condon and Shortley dipole transition strengths $S_{a b}$, statistical weights $g_{a}$ and a dimensionless quantity $S$ (of order unity) that incorporates all dependence on polarization directions. We use the formulas

$$
\begin{aligned}
\left\{\alpha_{11}(P, P)\right\} & =\frac{\left(e a_{0}\right)^{2}}{\hbar \Delta} \frac{S_{13}}{3 g_{1}} \\
\left\{\alpha_{22}(S, S)\right\} & =\frac{\left(e a_{0}\right)^{2}}{\hbar \Delta} \frac{S_{23}}{3 g_{2}} \\
\left\{\left|\alpha_{12}(P, S)\right|^{2}\right\} & =\left\{\alpha_{11}(P, P)\right\} \times\left\{\alpha_{22}(S, S)\right\} \mathcal{S}_{12}(S, P) \\
& =\frac{\left(e a_{0}\right)^{4}}{(\hbar \Delta)^{2}} \frac{S_{13}}{3 g_{1}} \times \frac{S_{23}}{3 g_{2}} S_{12}(S, P)
\end{aligned}
$$

and the Sommerfeld fine structure constant $\alpha=e^{2} / \hbar c$ to write

$$
\begin{aligned}
& K_{P}(z)=\left[2 \pi \alpha a_{0}^{2}\right] \mathcal{N}_{1}(z) \frac{\omega_{P}}{\Delta} \frac{S_{13}}{3 g_{1}} \\
& K_{S}(z)=\left[2 \pi \alpha a_{0}^{2}\right] \mathcal{N}_{2}(z) \frac{\omega_{S}}{\Delta} \frac{S_{23}}{3 g_{2}} \\
& \kappa_{S}(z)=\left[\frac{\pi \alpha^{2} c a_{0}^{4}}{2 \hbar}\right] \mathcal{N}^{\prime}(z) \frac{\omega_{S}}{\Delta^{2}} \frac{S_{13}}{3 g_{1}} \frac{S_{23}}{3 g_{2}} \mathcal{S}_{12}(S, P)
\end{aligned}
$$

The gain and dispersion parameters are expressible as

$$
\begin{aligned}
& g(z)=\left[\frac{8 \pi^{2} \alpha^{2} a_{0}^{4}}{\hbar}\right] \mathcal{N}(z) \frac{\omega_{S}}{(\Delta)^{2}} F_{P}(0) \frac{\tau_{R}}{T_{P}} \frac{S_{13}}{3 g_{1}} \frac{S_{23}}{3 g_{2}} S_{12}(S, P) \\
& \tau_{d i s}=\left[\frac{\hbar}{2 \pi \alpha c a_{0}^{2}}\right] \frac{T_{P}}{\tau_{R} F_{P}(0)} \frac{\mathcal{N}_{1}}{\mathcal{N}} \frac{\omega_{P}}{\omega_{S}} \frac{3 g_{2}}{S_{23}} \frac{1}{S_{12}(S, P)} \\
& \zeta=\frac{K_{S}(z)}{K_{P}(z)}=\frac{\mathscr{N}_{2}}{\mathcal{N}_{1}} \frac{\omega_{S}}{\omega_{P}} \frac{g_{1}}{g_{2}} \frac{S_{23}}{S_{13}}
\end{aligned}
$$

Note that the pump dispersion time $\tau_{\text {dis }}$ is independent of the detuning $\Delta$ and of distance $z$ (because the ratio $\mathscr{r}_{1} / \mathcal{N}$ is taken as uniform). 


\section{$\S 4.4$ Oscillator Strengths}

An alternative choice for expressing atomic dipole transition moments is the (absorption) oscillator strengths $f_{a b}$,

$$
f_{13}=\left[\frac{4 \pi a_{0}}{\alpha}\right] \frac{1}{\lambda_{P}} \frac{S_{13}}{3 g_{1}}, \quad f_{23}=\left[\frac{4 \pi a_{0}}{\alpha}\right] \frac{1}{\lambda_{S}} \frac{S_{23}}{3 g_{2}}
$$

Using these we write

$$
\begin{aligned}
& K_{P}(z)=\left[\pi \alpha^{2} c a_{0}\right] \frac{\mathcal{N}_{1}(z)}{\Delta} f_{13} \\
& K_{S}(z)=\left[\pi \alpha^{2} c a_{0}\right] \frac{\mathcal{N}_{2}(z)}{\Delta} f_{23} \\
& \kappa_{S}(z)=\left[\frac{\alpha^{4} c^{2} a_{0}^{2}}{16 \hbar}\right] \frac{\mathcal{N}(z)}{\Delta^{2}} \lambda_{P} f_{23} f_{13} S_{12}(S, P)
\end{aligned}
$$

and hence

$$
\begin{aligned}
& g(z)=\left[2 \pi^{2} \alpha^{4} c^{2} a_{0}{ }^{2}\right] \frac{\mathcal{N}(z)}{\Delta^{2}} \frac{F_{P}(0)}{\hbar \omega_{P}} \frac{\tau_{R}}{T_{P}} f_{13} f_{23} \mathcal{S}_{12}(S, P) \\
& \tau_{d i s}=\left[\frac{1}{\pi \alpha^{2} c a_{0}}\right] \frac{T_{P}}{\tau_{R}} \frac{\hbar \omega_{P}}{F_{P}(0)} \frac{\mathcal{N}_{1}}{\mathcal{N}} \frac{1}{f_{23}} \frac{1}{S_{12}(S, P)} \\
& \zeta=\frac{\mathcal{N}_{2}}{\mathcal{N}_{1}} \frac{f_{23}}{f_{13}}
\end{aligned}
$$

Note the appearance in these formulas $\sigma_{i}{ }^{\circ}$ the total photon fluence $F_{P}(0) / \hbar \omega_{P}$. 


\section{$\S 4.5$ Rabi Frequencies}

To facilitate interpretation of coherent excitation we define the instantaneous pump Rabi frequency $\Omega_{P}$ for the $1 \longmapsto 3$ transition,

$$
\Omega_{P}(z, t)=\mathscr{E}_{P}(z, t) \frac{d_{13}}{\hbar}
$$

A satisfactory definition of the pump Rabi frequency when degeneracy is present is

$$
\left|\Omega_{P}(z, t)\right|^{2}=\left[\frac{2 \alpha^{2} a_{0}}{\hbar}\right] \lambda_{P} f_{13} I_{P}(z, t)=\left[\frac{8 \pi \alpha\left(a_{0}\right)^{2}}{\hbar}\right] \frac{S_{13}}{3 g_{1}} I_{P}(z, t)
$$

where $f_{13}$ is the (absorption) oscillator strength and $S_{13}$ is the dipole transition strength. We introduce the incident mean pump Rabi frequency

$$
|\bar{\Omega}|^{2}=\left[\frac{8 \pi \alpha a_{0}^{2}}{\hbar}\right] \frac{S_{13}}{3 g_{1}} \frac{F_{P}(0)}{T_{P}}
$$

so that the formulas for propagation and dispersion parameters become

$$
\begin{aligned}
& g(z)=\left[\frac{2 \pi \alpha a_{0}^{2}}{2}\right] \mathcal{N}(z) \omega_{S} \tau_{R} \frac{|\bar{\Omega}| 2}{\Delta^{2}} \frac{S_{23}}{3 g_{2}} S_{12}(S, P) \\
& \tau_{d i s}=\tau_{R} \frac{4}{\left(\tau_{R} \bar{\Omega}\right)^{2}} \frac{\mathcal{N}_{1}}{\mathcal{N}^{2}} \frac{\omega_{P}}{w_{S}} \frac{g_{2}}{g_{1}} \frac{S_{13}}{S_{23}} \frac{1}{S_{12}(S, P)}
\end{aligned}
$$

or

$$
\begin{aligned}
& g(z)=\left[\frac{2 \pi \alpha a_{0}{ }^{2}}{4}\right] \mathscr{N}(z) \tau_{R} \frac{|\bar{\Omega}| 2}{\Delta^{2}} f_{23} S_{12}(S, P) \\
& \tau_{d i s}=\tau_{R} \frac{4}{\left(\tau_{R} \bar{\Omega}\right)^{2}} \frac{\mathcal{N}_{1}}{\mathcal{N}} \frac{f_{13}}{f_{23}} \frac{1}{S_{12}(S, P)}
\end{aligned}
$$


2 Aug 91

dimers -25

Raman Propagation Equations

$$
\begin{aligned}
& \frac{\partial}{\partial z} \mathscr{E}_{S}(z, t)=+i \mathscr{K}_{S} \mathscr{E}_{S}(z, t) \\
& \quad-\kappa_{S} \int_{-\infty}^{\infty} d t^{\prime} \mathscr{G}\left(t-t^{\prime}\right) w\left(z, t^{\prime}\right) \mathscr{F}_{P}\left(z, t^{\prime}\right)^{*} \mathscr{E}_{S}\left(z, t^{\prime}\right) \mathscr{F}_{P}(z, t)
\end{aligned}
$$

,

$$
\begin{aligned}
& \frac{\partial}{\partial z} \mathscr{E}_{P}(z, t)=+i \mathscr{K}_{P} \mathscr{E}_{P}(z, t) \\
& \quad+\kappa_{P} \int_{-\infty}^{\infty} d t^{\prime} \mathscr{G}\left(t-t^{\prime} w\left(z, t^{\prime}\right) \mathscr{F}_{P}\left(z, t^{\prime}\right) \mathcal{E}_{S}\left(z, t^{\prime}\right)^{*} \mathscr{F}_{S}(z, t)\right.
\end{aligned}
$$

where

$$
\mathscr{F}_{\lambda}(i)=\left[1+\frac{i}{\Delta} \frac{\partial}{\partial t}+\ldots\right] \mathscr{E}_{\lambda}(t)
$$


2 Aug 91

dimers -26

Raman Propagation Parameters

Dispersive

$$
\begin{aligned}
& \mathscr{K}_{P} \mathcal{E}_{P}=\frac{2 \pi \omega_{P}}{c} \mathcal{N}_{1}\left\{\alpha_{11}(P, P)\right\} \mathscr{F}_{P}=\left[2 \pi \alpha a_{0}{ }^{2}\right] \mathcal{N}_{1} \frac{\omega_{P}}{\Delta} \frac{S_{13}}{3 g_{1}} \mathscr{T}_{P} \\
& \mathscr{K}_{S} \mathcal{E}_{S}=\frac{2 \pi \omega_{S}}{c} \mathcal{N}_{2}\left\{\alpha_{22}(S, S)\right\} \mathscr{F}_{S}=\left[2 \pi \alpha a_{0}{ }^{2}\right] \mathcal{N}_{2} \frac{\omega_{S}}{\Delta} \frac{S_{23}}{3 g_{2}} \mathscr{F}_{S}
\end{aligned}
$$

Raman gain

$$
\begin{aligned}
& \kappa_{S}=\frac{\pi \omega_{S}}{2 \hbar c} \mathcal{N}\left\{\left|\alpha_{12}(P, S)\right|^{2}\right\}=\left[\frac{\pi \alpha^{2} c a_{0}^{4}}{2 \hbar}\right] \mathcal{N} \frac{\omega_{S}}{(\Delta)^{2}} \frac{S_{13}}{3 g_{1}} \frac{S_{23}}{3 g_{2}} \mathcal{S}_{12}(S, P) \\
& \kappa_{P}=\frac{\omega_{P}}{\omega_{S}} \kappa_{S}
\end{aligned}
$$

The gain coefficient

$$
\begin{aligned}
& g=2 \kappa_{S}\left|\mathscr{O}_{P}\right|^{2} \tau_{R} \\
& g=2\left[\frac{\pi \alpha^{2} c a_{0}^{4}}{2 \hbar}\right] \mathcal{N} \frac{\omega_{S}}{(\Delta)^{2}} F_{P}(0) \frac{\tau_{R}}{T_{P}} \frac{S_{13}}{3 g_{1}} \frac{S_{23}}{3 g_{2}} \mathcal{S}_{12}(S, P) \\
& {\left[\frac{\mathscr{K}_{P}}{g}\right] \mathscr{E}_{P}=\left[\frac{4 \hbar}{\alpha c a_{0}^{2}}\right] \frac{\mathcal{N}_{1}}{\mathcal{N}} \frac{\omega_{P}}{\omega_{S}} \frac{T_{P} \Delta}{\tau_{R} F_{P}(0)} \frac{3 g_{2}}{S_{23}} \frac{1}{S_{12}} \mathscr{F}_{P}}
\end{aligned}
$$

Introduce incident mean pump Rabi frequency

$$
\left|\bar{\Omega}_{P}\right|^{2}=\left[\frac{8 \pi \alpha a_{0}^{2}}{\hbar}\right] \frac{S_{13}}{3 g_{1}} \frac{F_{P}(0)}{T_{P}}
$$

so that

$$
\begin{aligned}
& g=\left[2 \pi \alpha a_{0}^{2}\right] \mathscr{N} \omega_{S} \tau_{R} \frac{\left|\bar{\Omega}_{P}\right|^{2}}{\Delta^{2}} \frac{S_{23}}{3 g_{2}} \mathcal{S}_{12}(S, P) \\
& {\left[\frac{\mathscr{K}_{P}}{g}\right] \mathcal{E}_{P}=4 \frac{\mathcal{N}_{1}}{\mathscr{N}^{2}} \frac{\omega_{P}}{\omega_{S}} \frac{\Delta^{2}}{\left|\bar{\Omega}_{P}\right|^{2}} \frac{1}{\tau_{R} \Delta} \frac{g_{2}}{g_{1}} \frac{S_{13}}{S_{23}} \frac{1}{S_{12}} \mathscr{F}_{P}}
\end{aligned}
$$


near square pulse

Fig. $1 a$

$$
\text { short relax }
$$

stokes $=$ pump

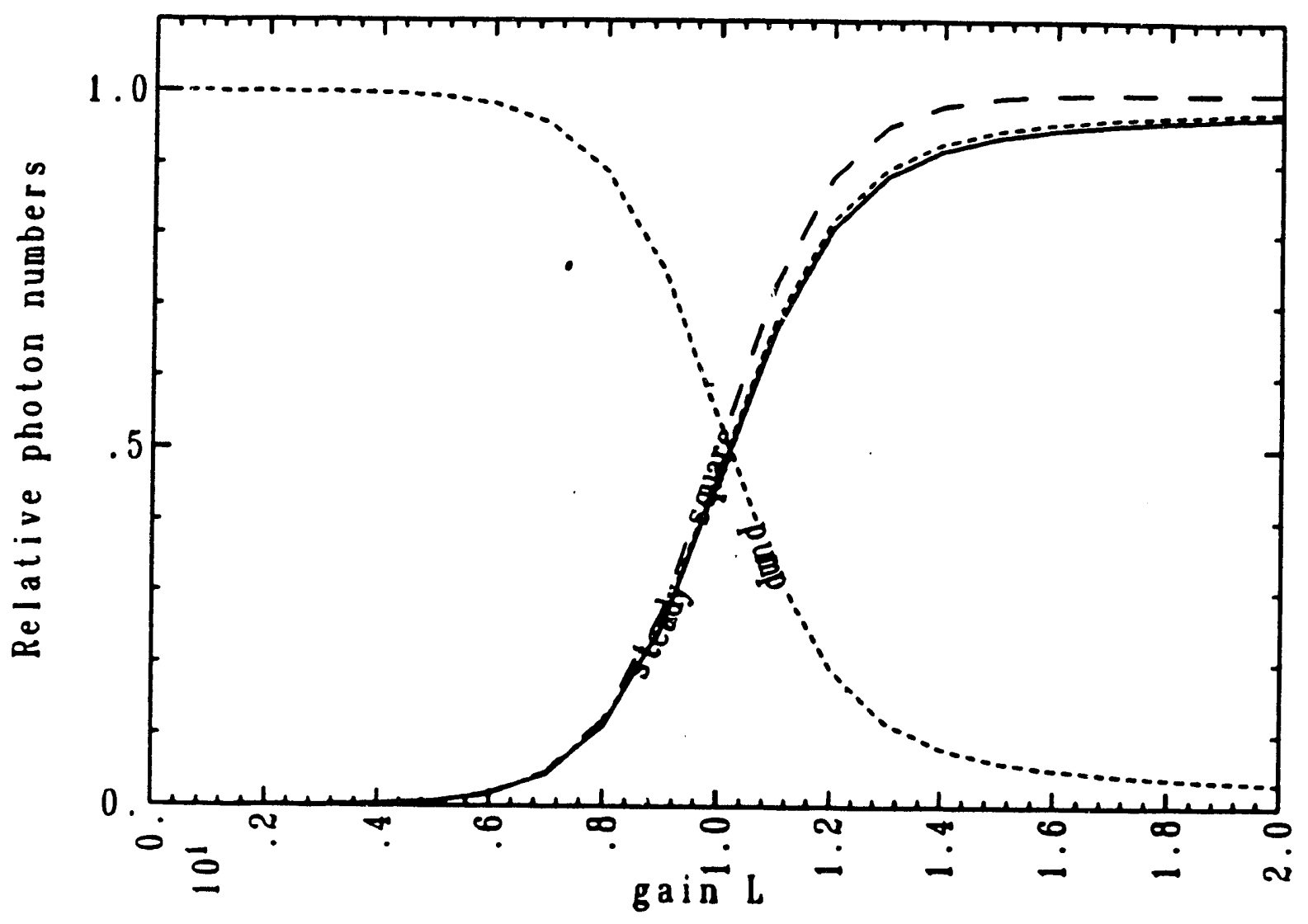




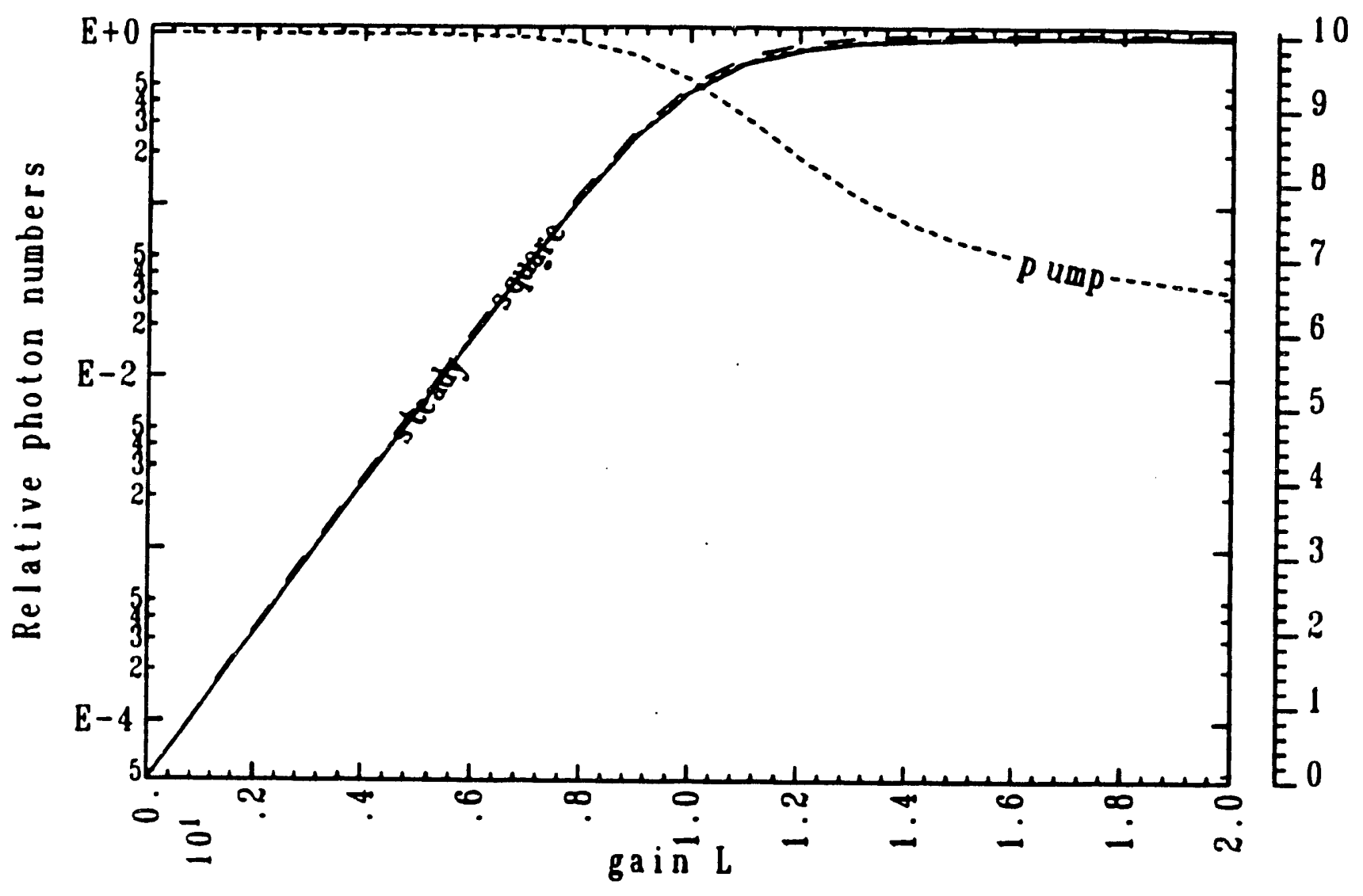



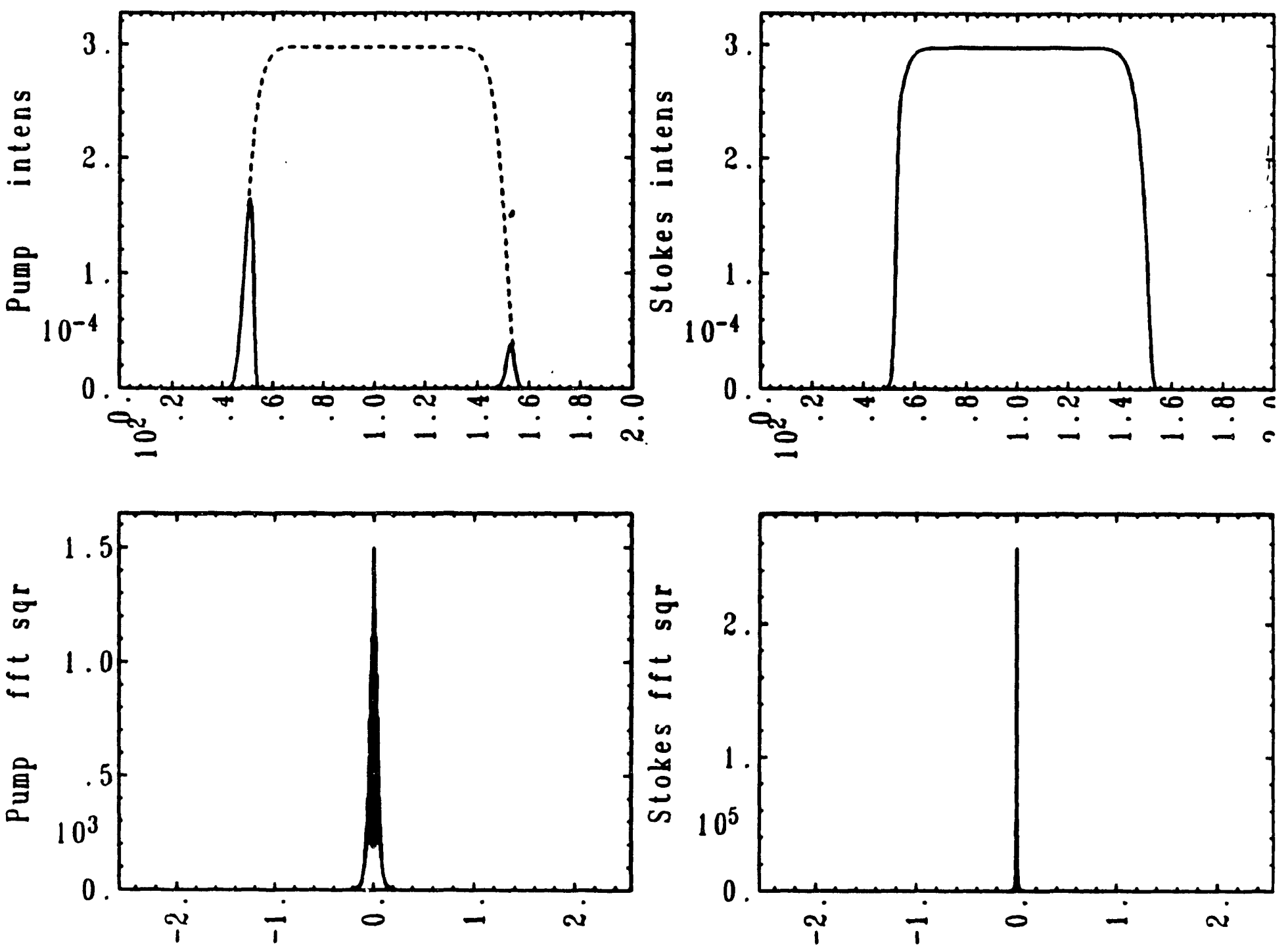


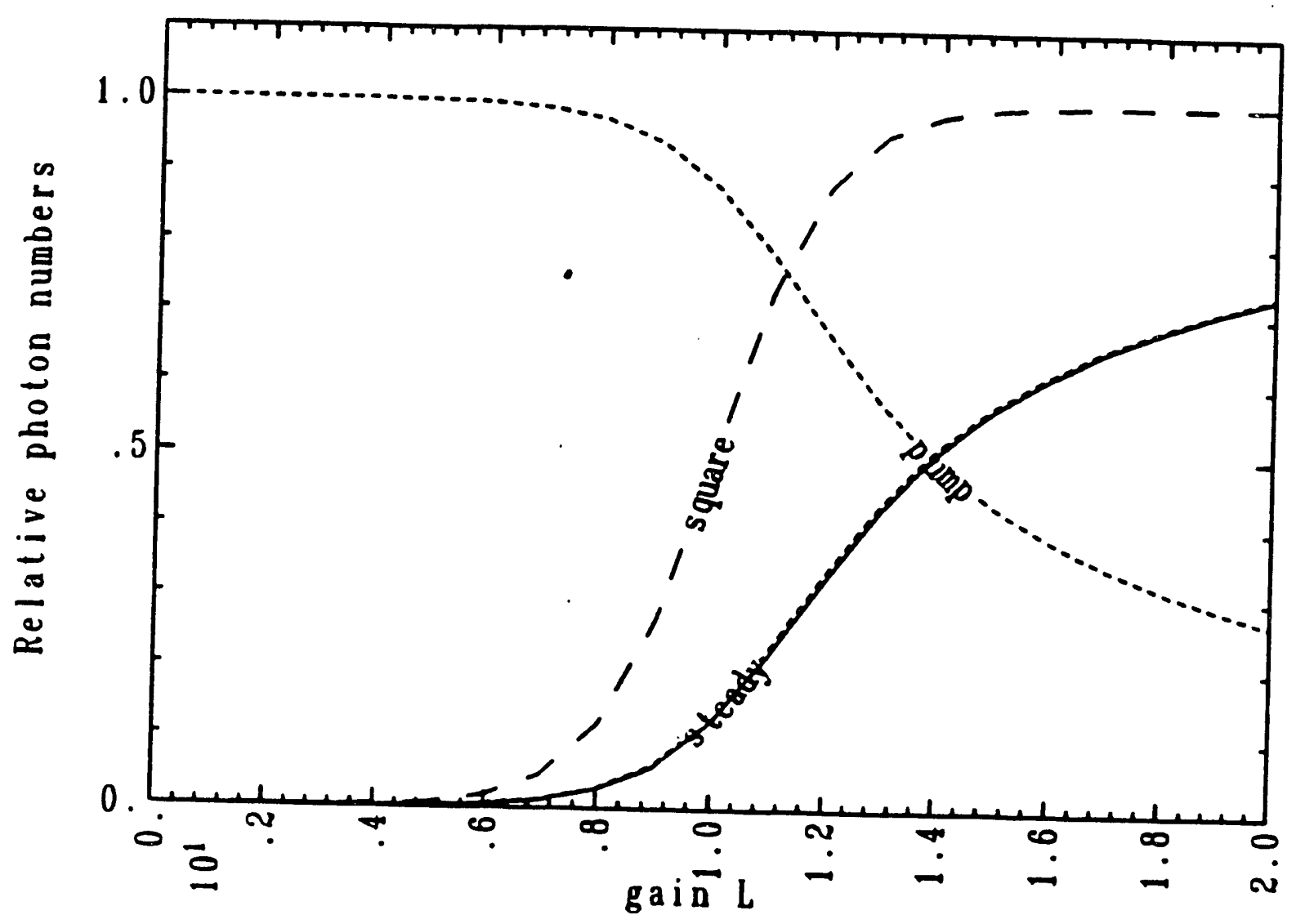




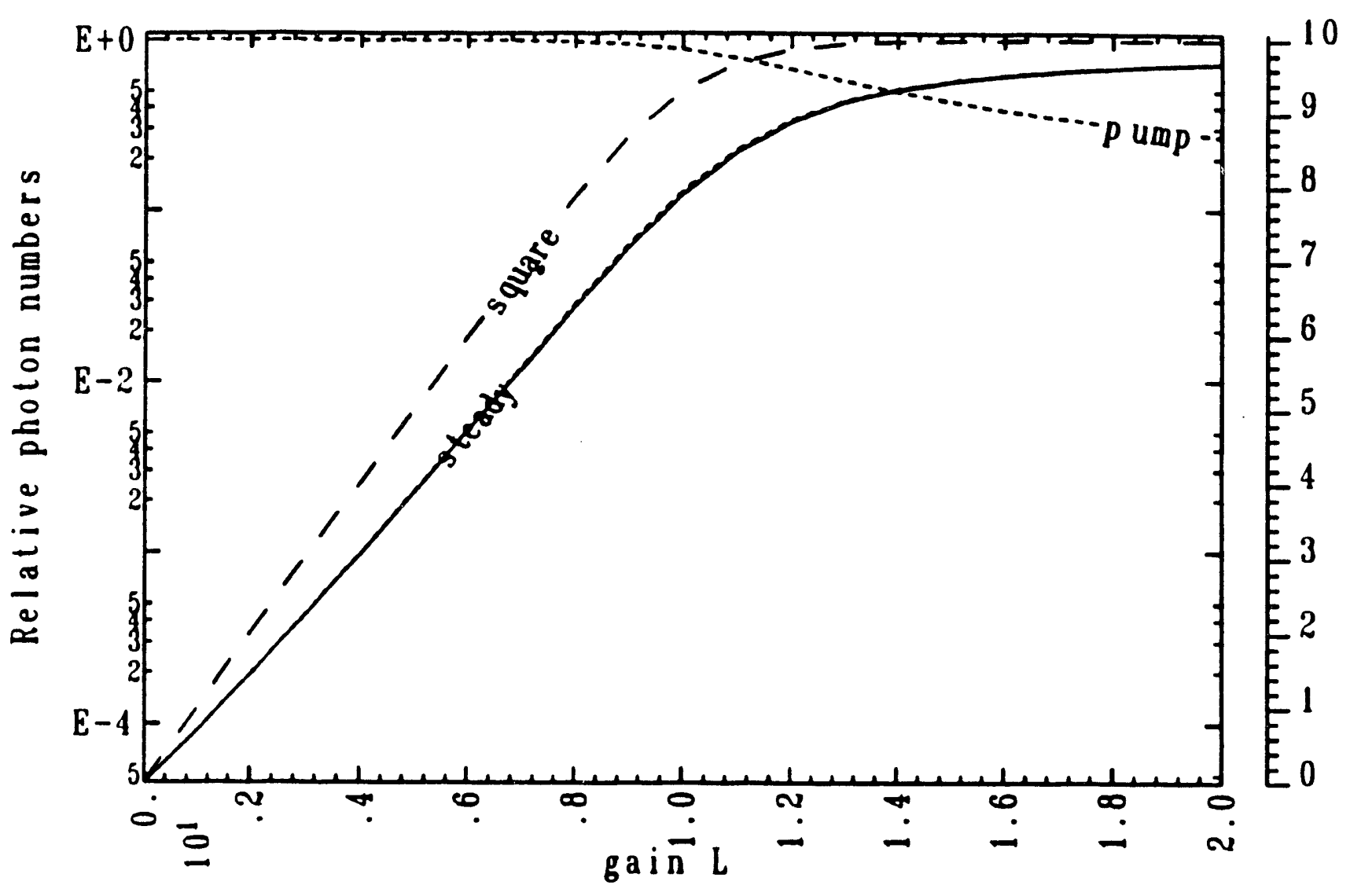


Shape

Fig. $2 c$

Gauss 2 pulse

short relax

stokes $=$ pump

Pump

at $z=2.00 E+01$

Stokes
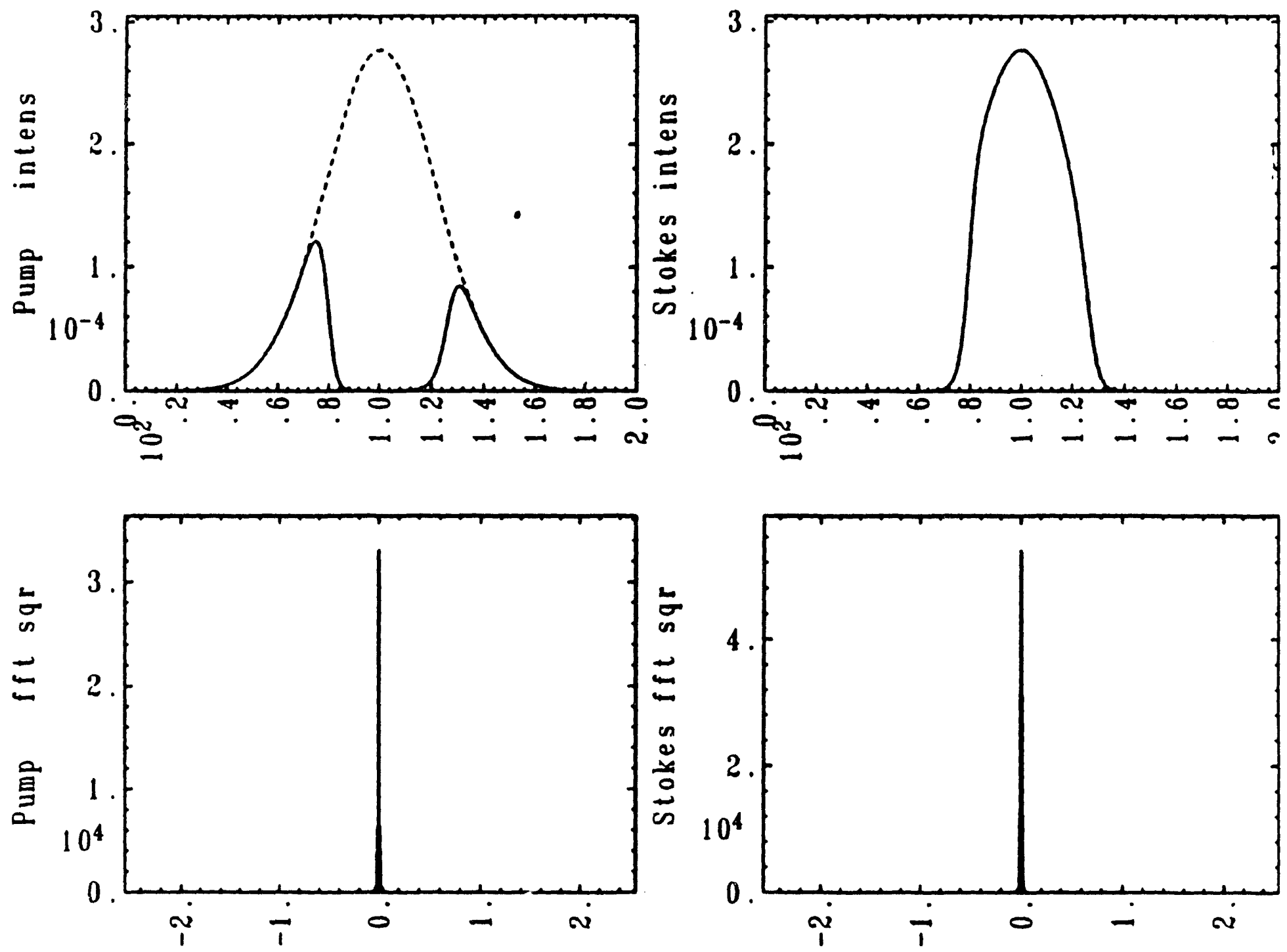

06-28-91 07:37:49h frame 16 gauss 2 pump = stokes 
near square pulse short relax

const stokes seed

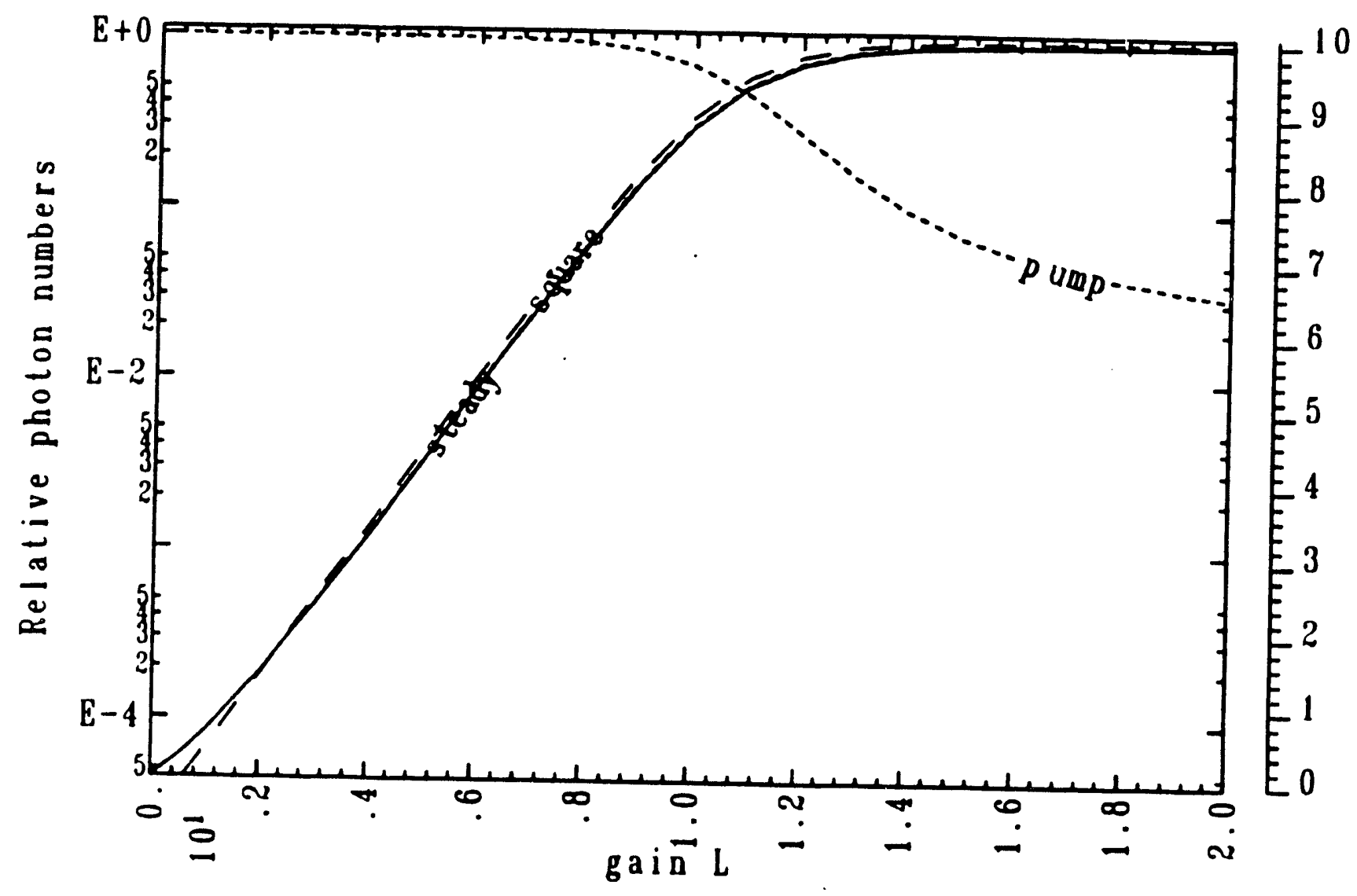


near square pulse with warble

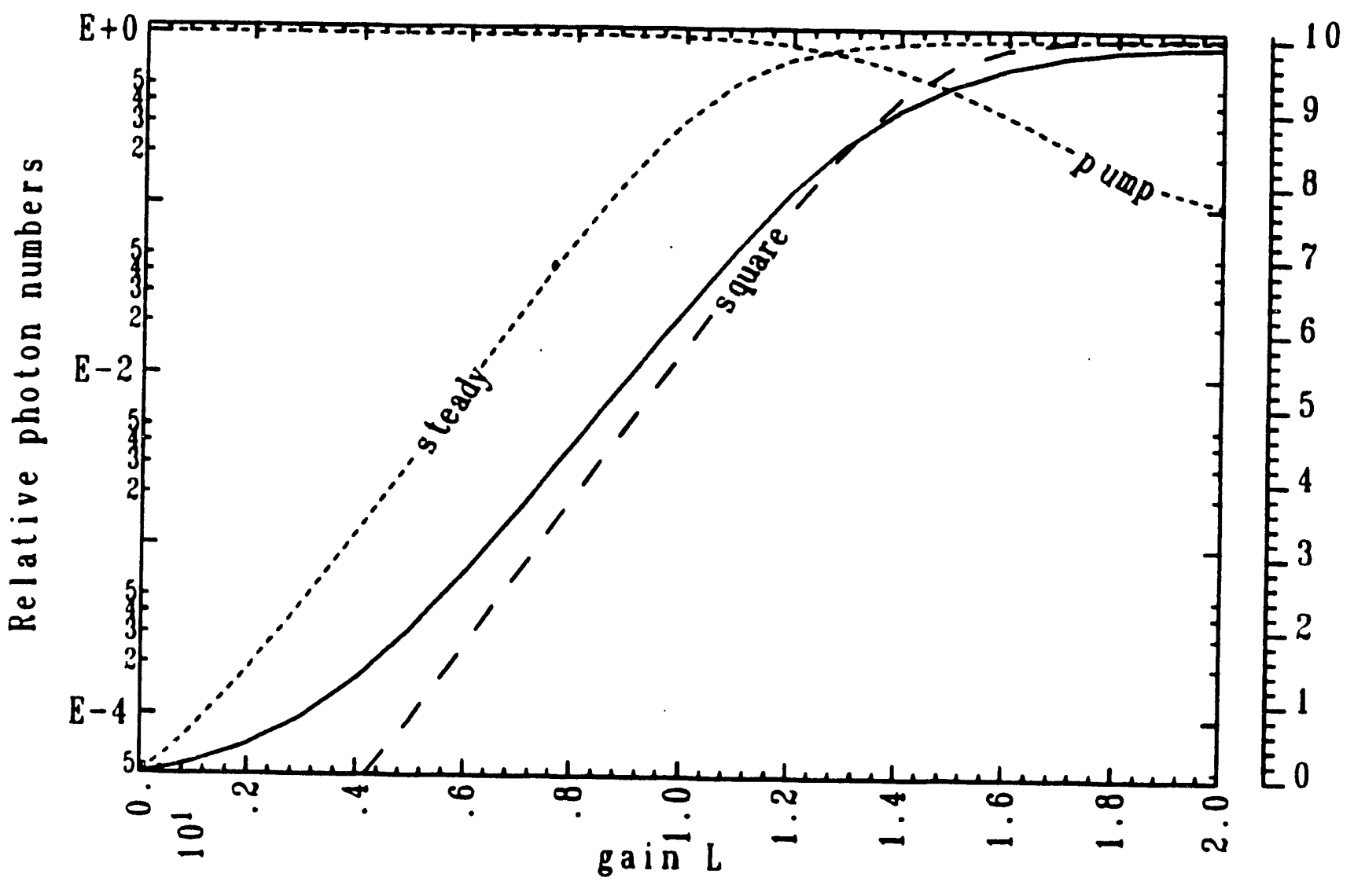


near square pulse with warble

Fig. $4 b$ short relax

const stokes seed

Pump

at $z=2.00 \mathrm{E}+01$

Stokes
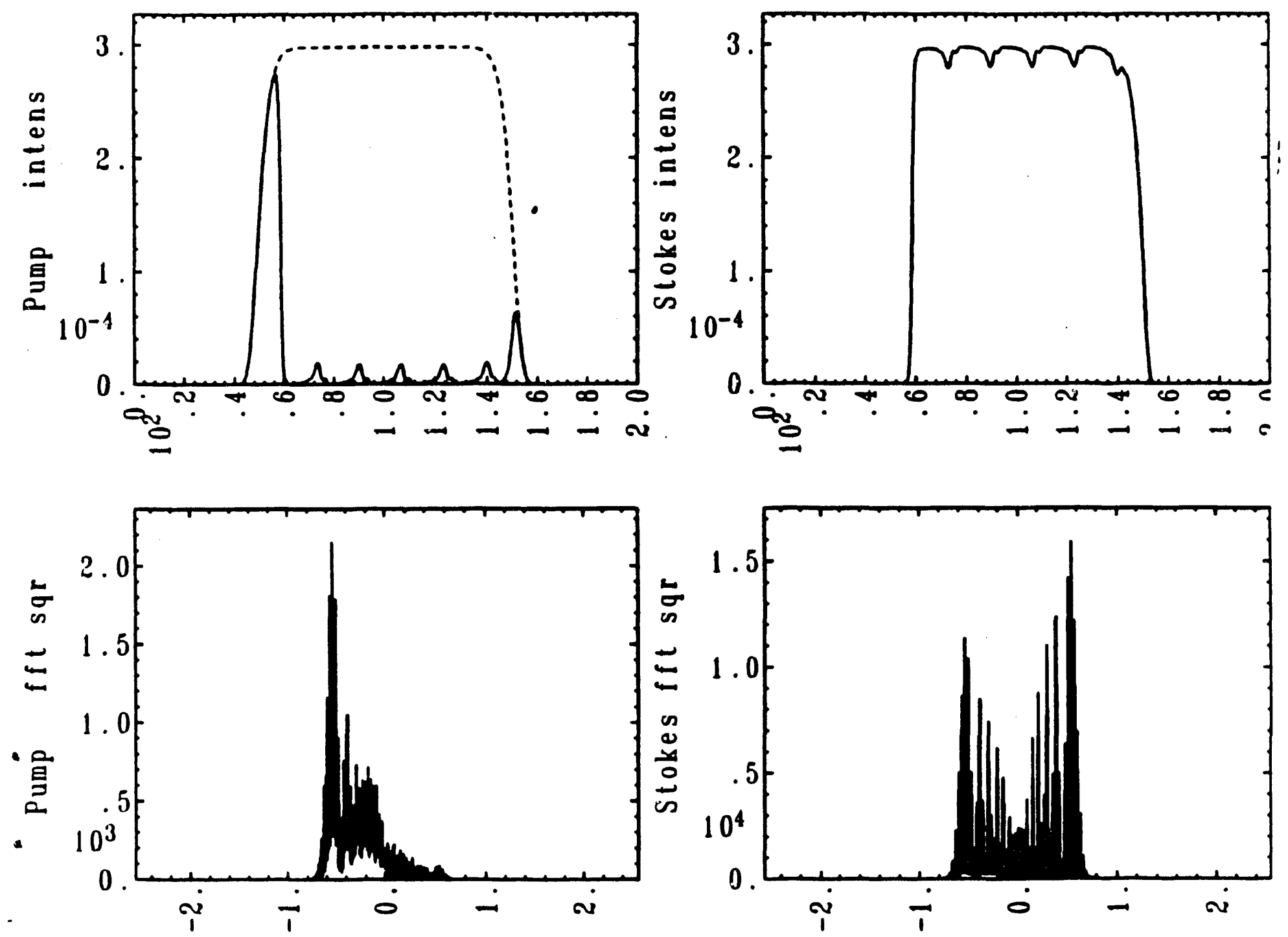
near square pulse with warble

Fig. $5 a$

short relax

noise stokes seed

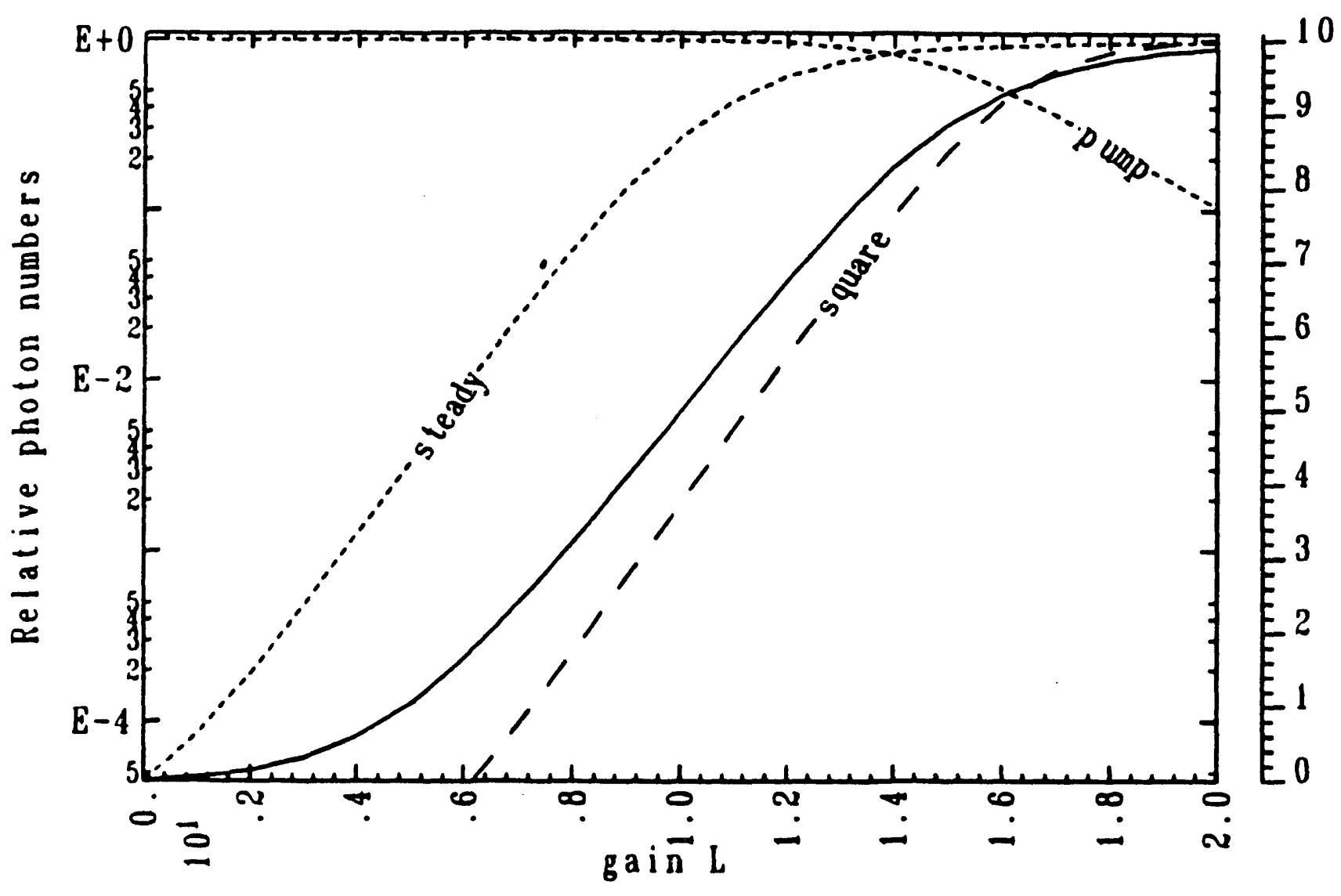




\section{Correlation}

near square pulse with warble

Fig. $5 b$

noise stokes seed

Pump

at $z=2.00 E+01$

Stokes
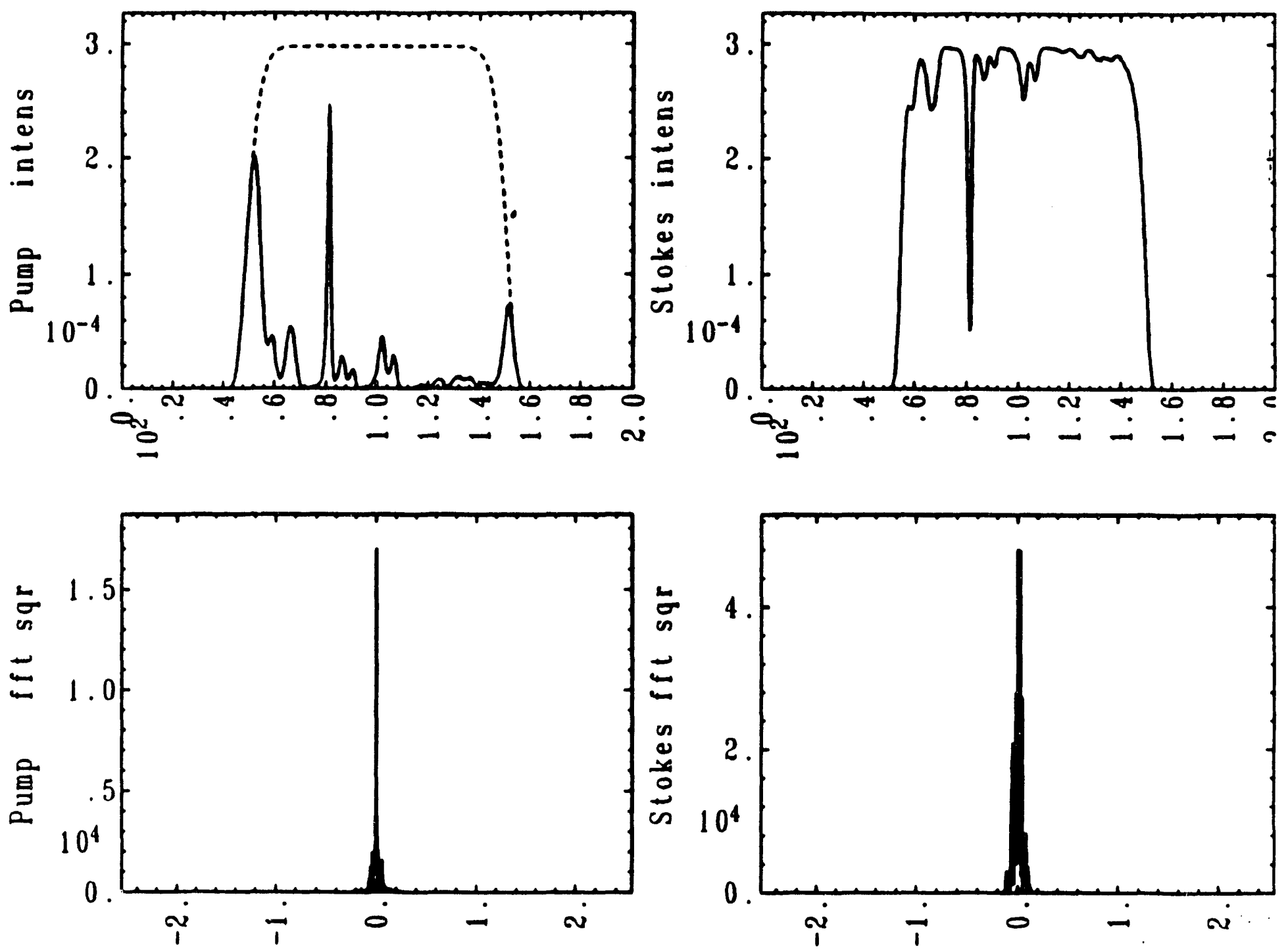
Phase

Fig. 6

near square pulse

short relax

noise stokes seed

ampl = noise phase $=$ noise
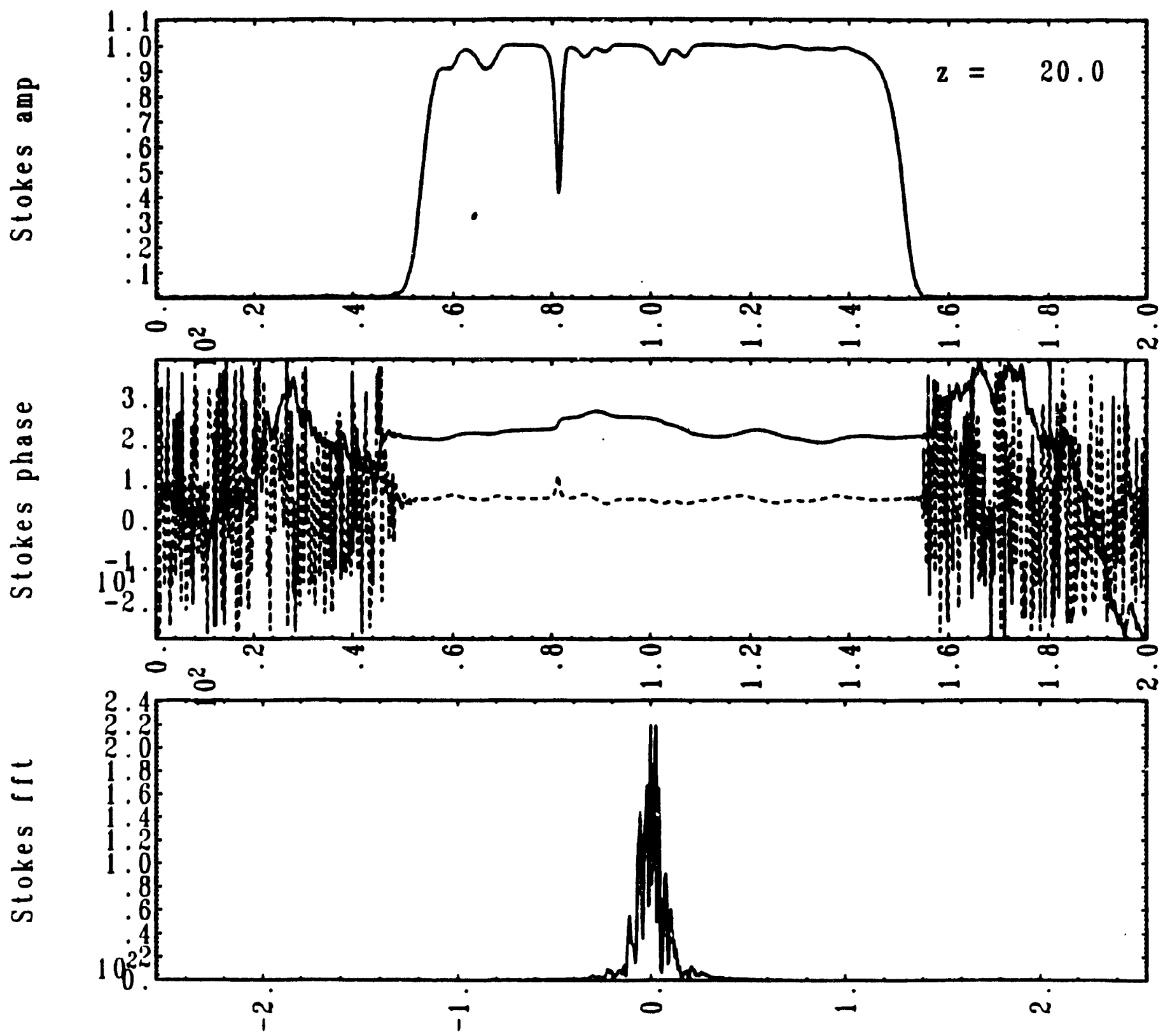

06-28-91 07:31:26h frame 13 bypergaus pump. ncise stokes 
Phase of Pump

near square pulse with warble

Fig. $7 a$

short relax

const stokes seed

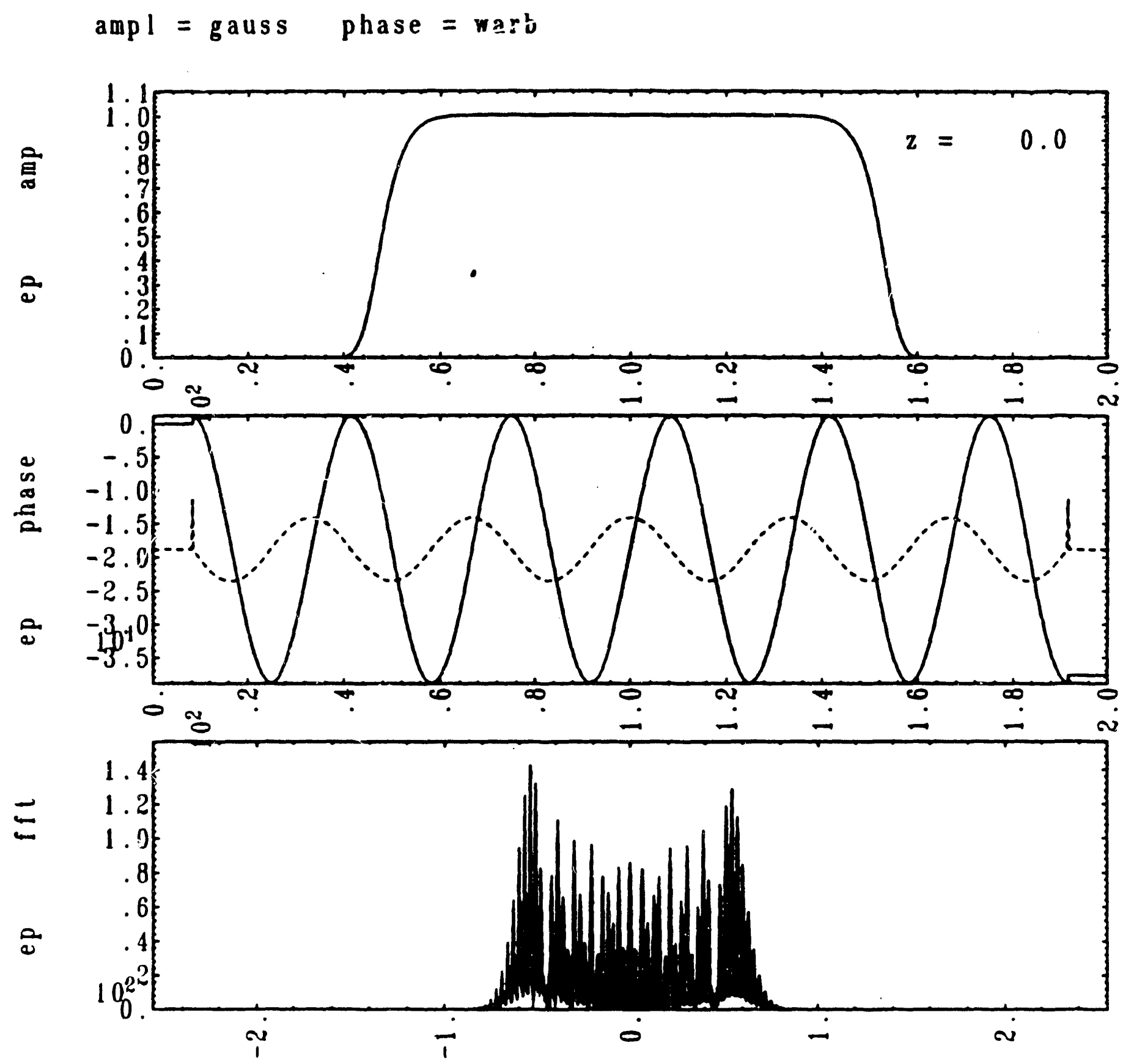

06-28-91 07:59:04b frame 2 warble punp, const stokes 
near square pulse with warble

ampl $=$ const phase $=$ zero
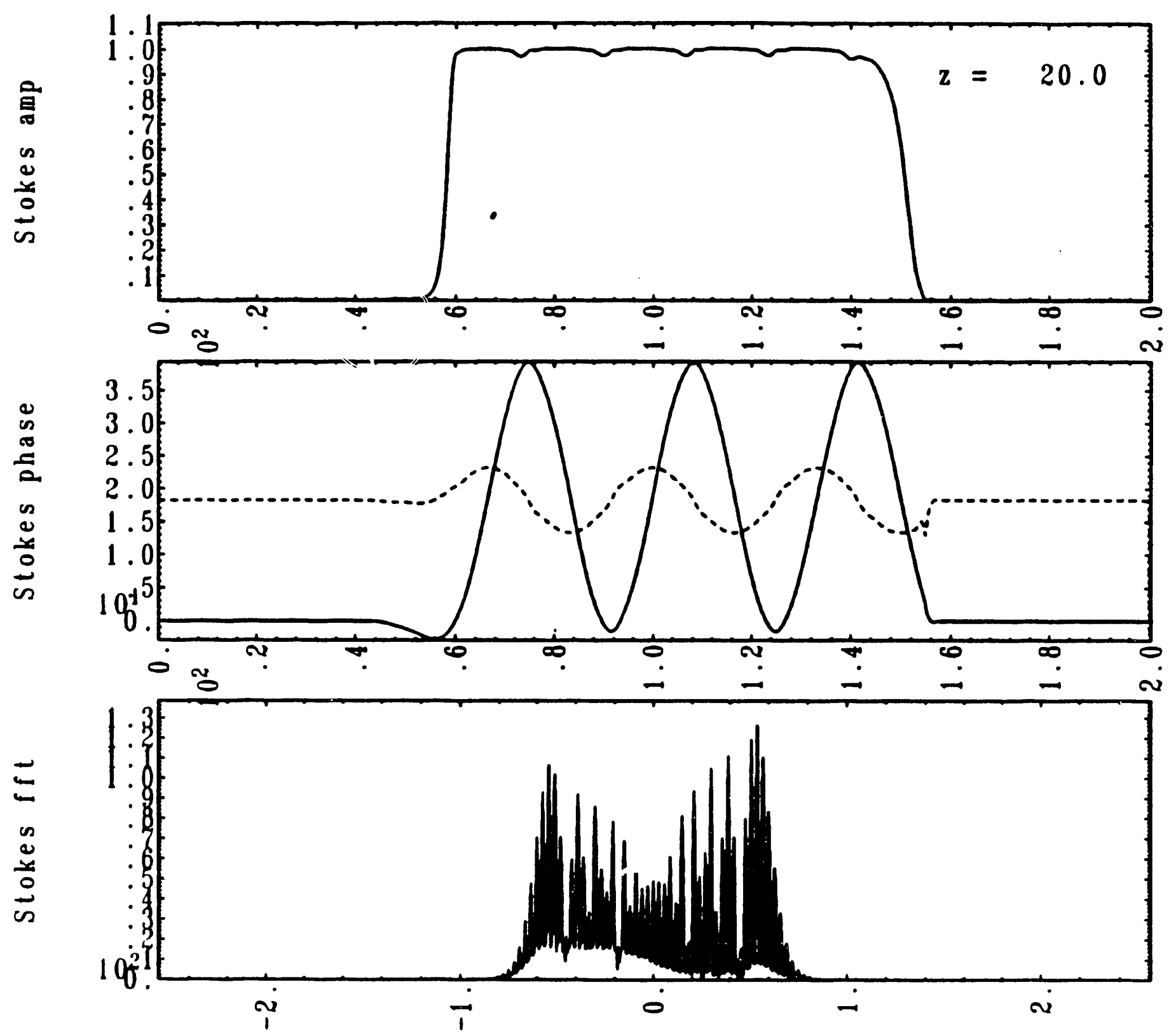

06-28-91 08:03:19b frame 6 warble punp, const stokes 


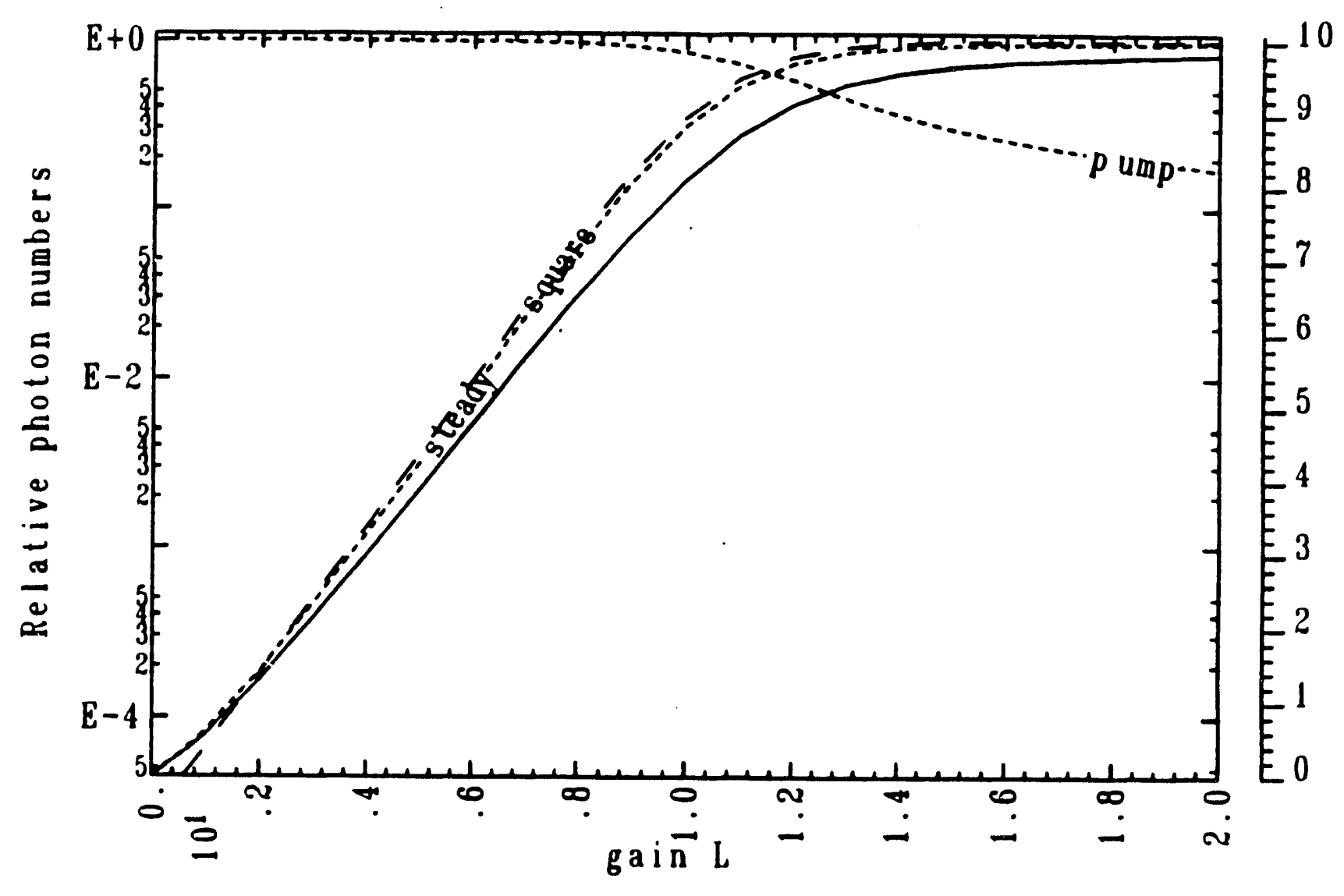



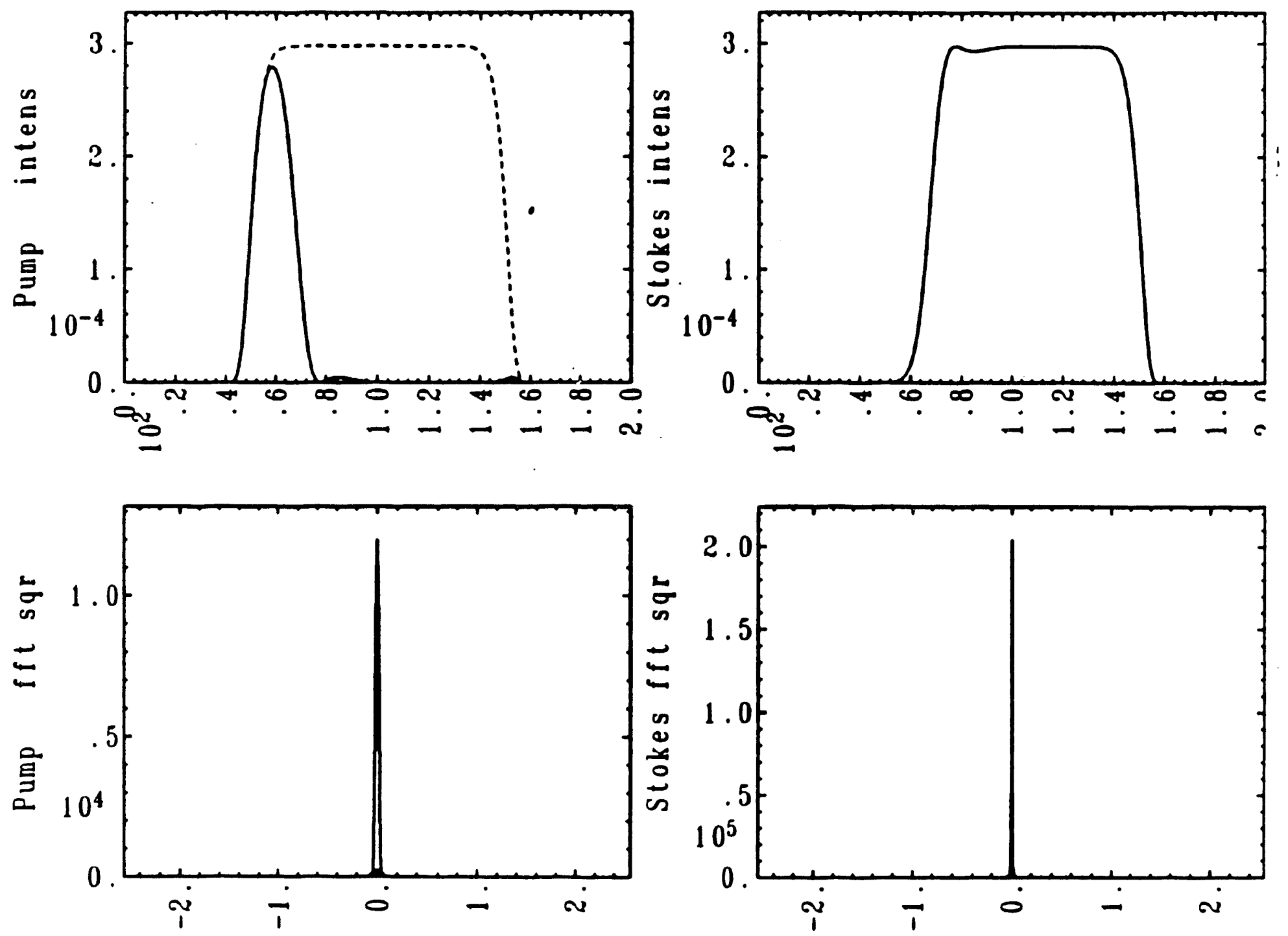
near square pulse

Fig. $9 a$

$T$ relax $=50$

const stokes seed

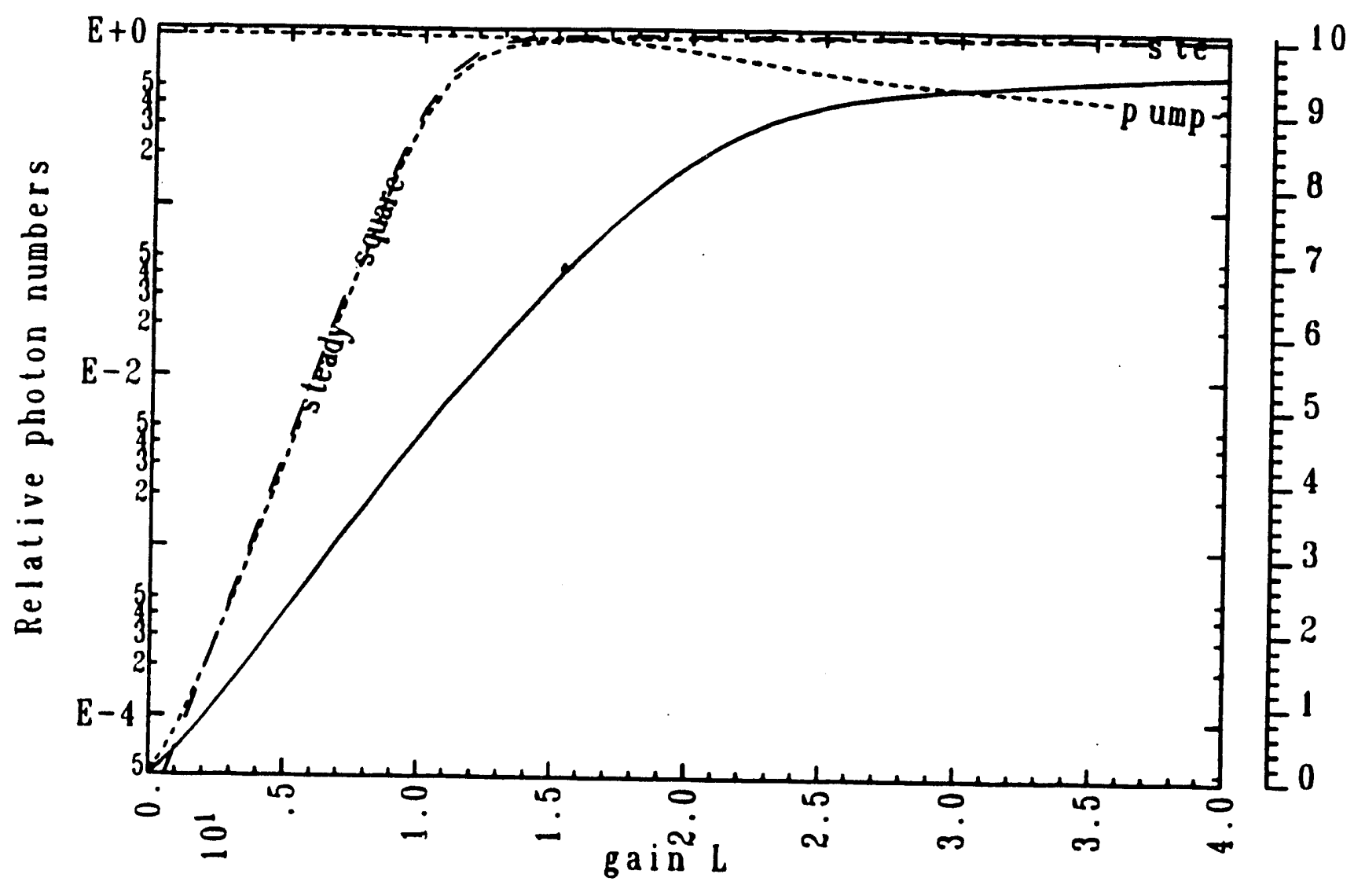


Transient

Fig. $9 b$

near square pulse

$T$ relax $=50, \quad Z=20$

const stokes seed

Pump

at $z=2.00 E+01$

Stokes
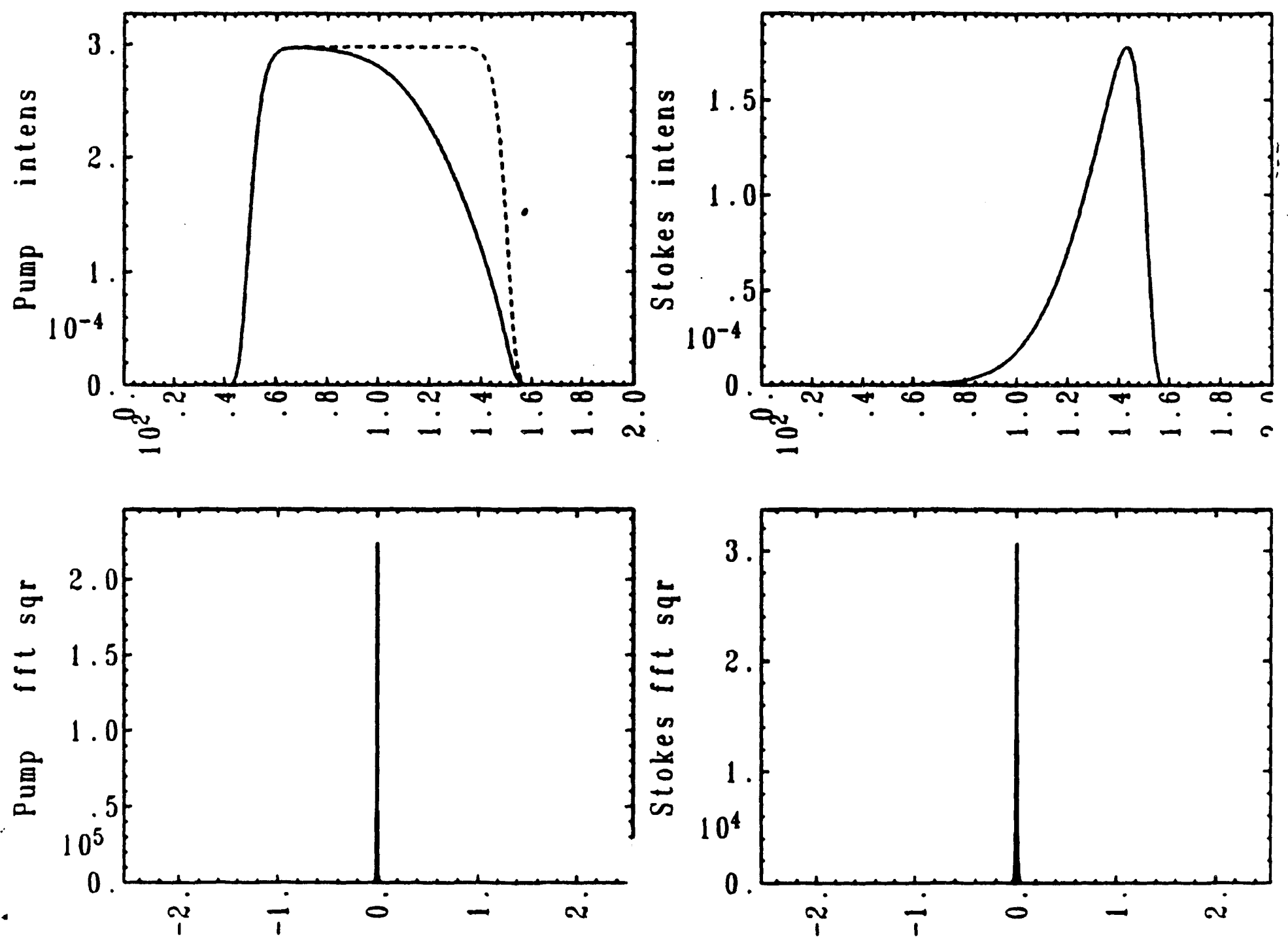

$06-28-91$ 
near square pulse

$T$ relax $=50, \quad Z=40$

const stokes seed

Pump

at $z=4.00 E+01$

Stokes
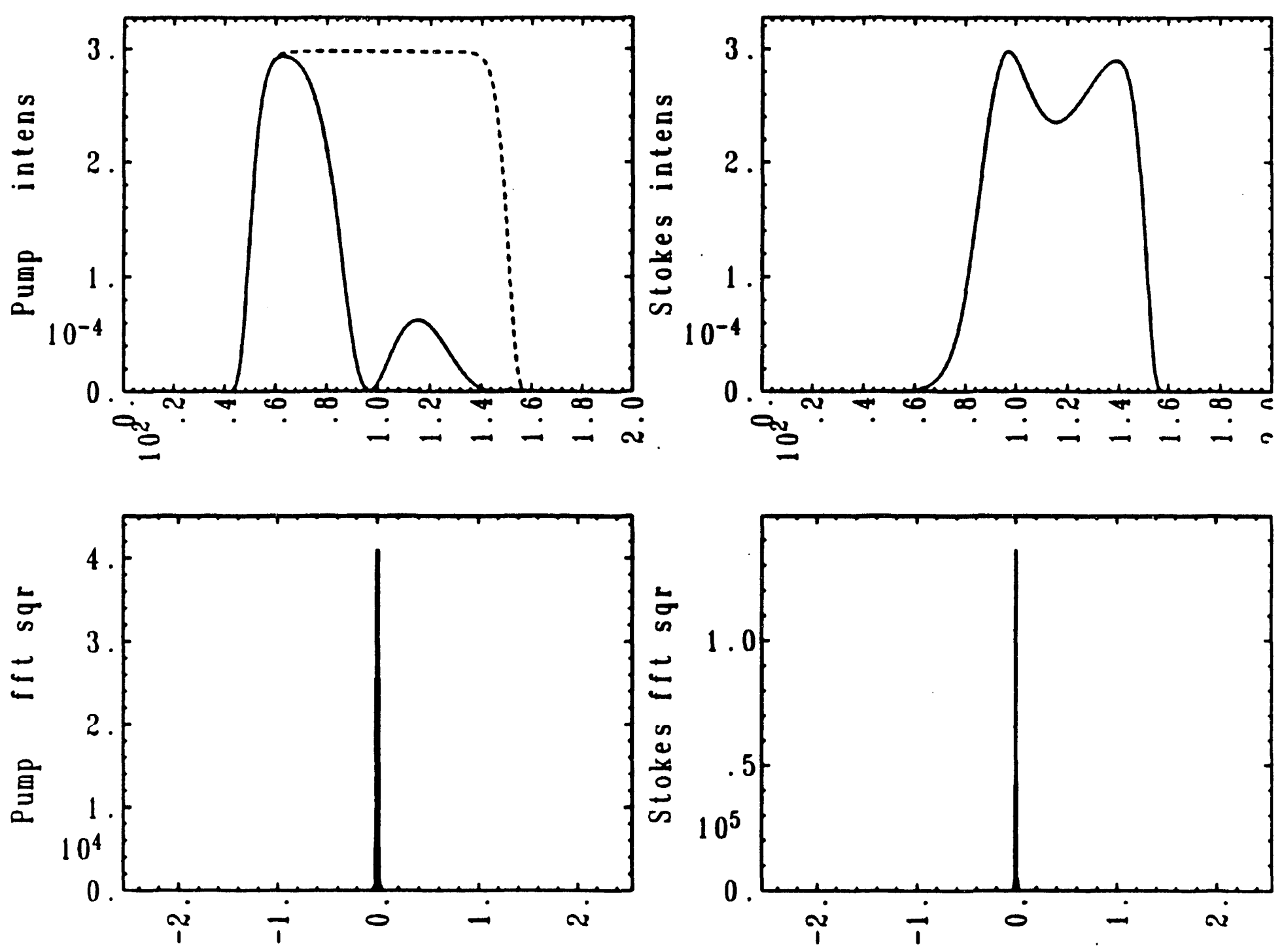
Dispersion

near square pulse

short relax

const stokes seed

$T$ disp $=5$

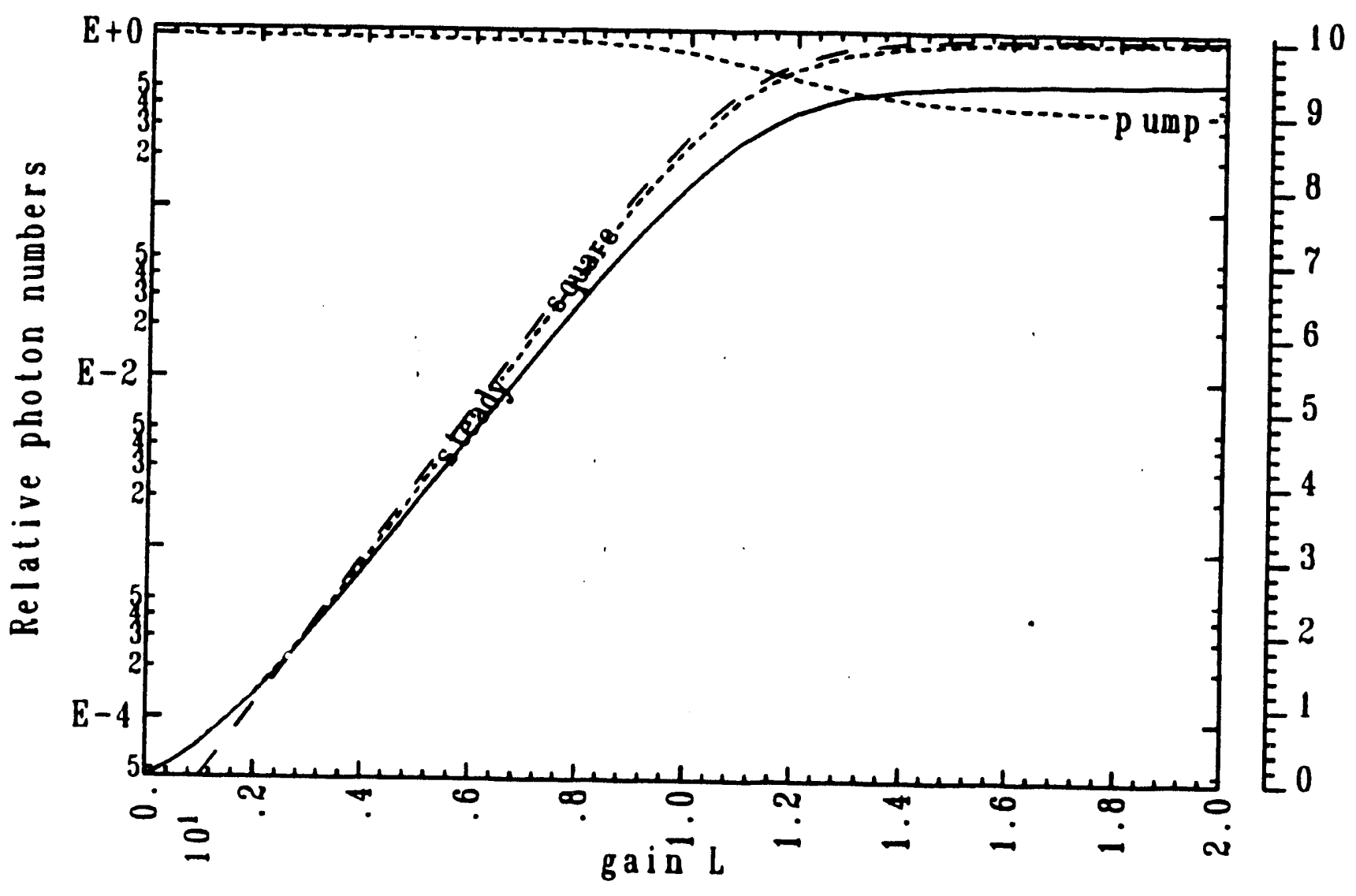




\section{Dispersion}

short relax

const stokes seed

$T$ disp $=5, \quad Z=5$ near square pulse

Fig. 10b
Stokes
at $z=5.00 \mathrm{E}+00$
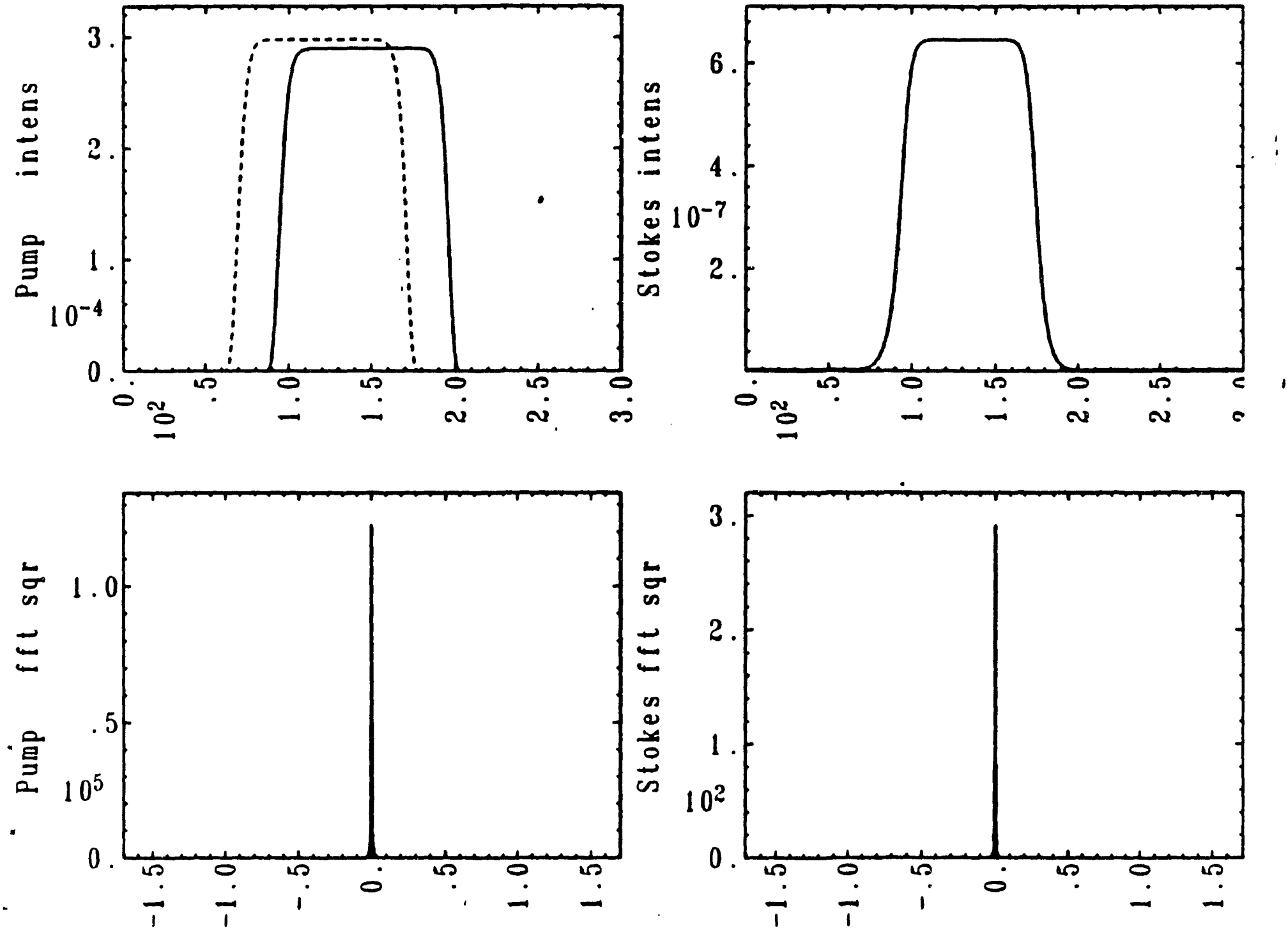
Dispersion

near square pulse

short relax

const stokes seed

$T$ disp $=5, \quad Z=10$
Fig. 10c

Pump

at $z=1.00 E+01$

Stokes
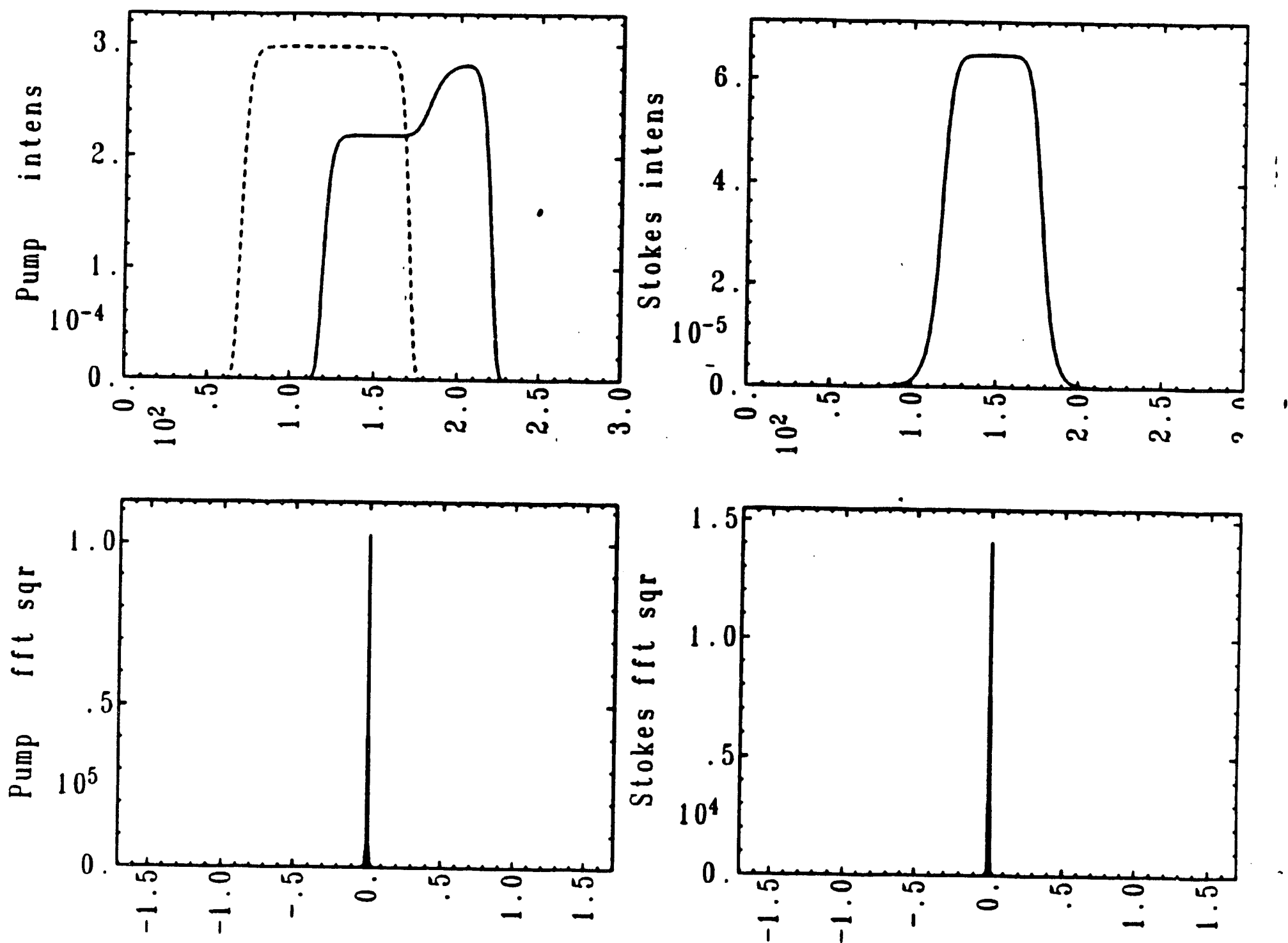
Dispersion

near square pulse

short relax

const stokes seed

$T$ disp $=5, \quad Z=20$
Fig. 10d

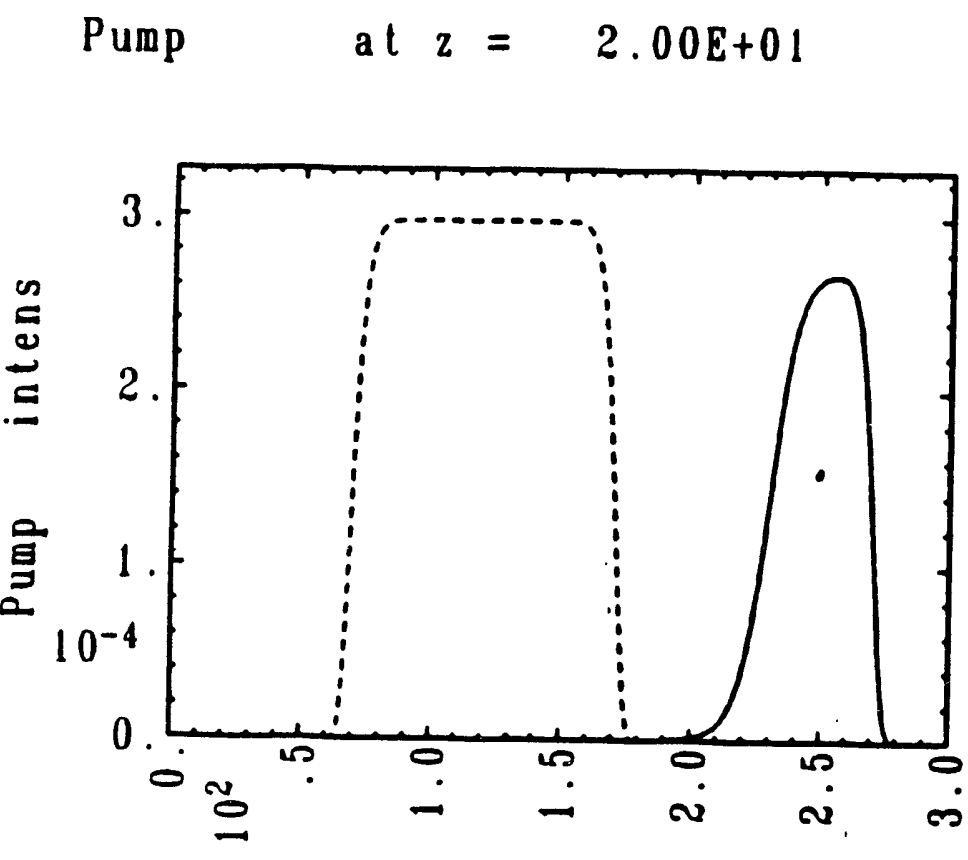

Stokes
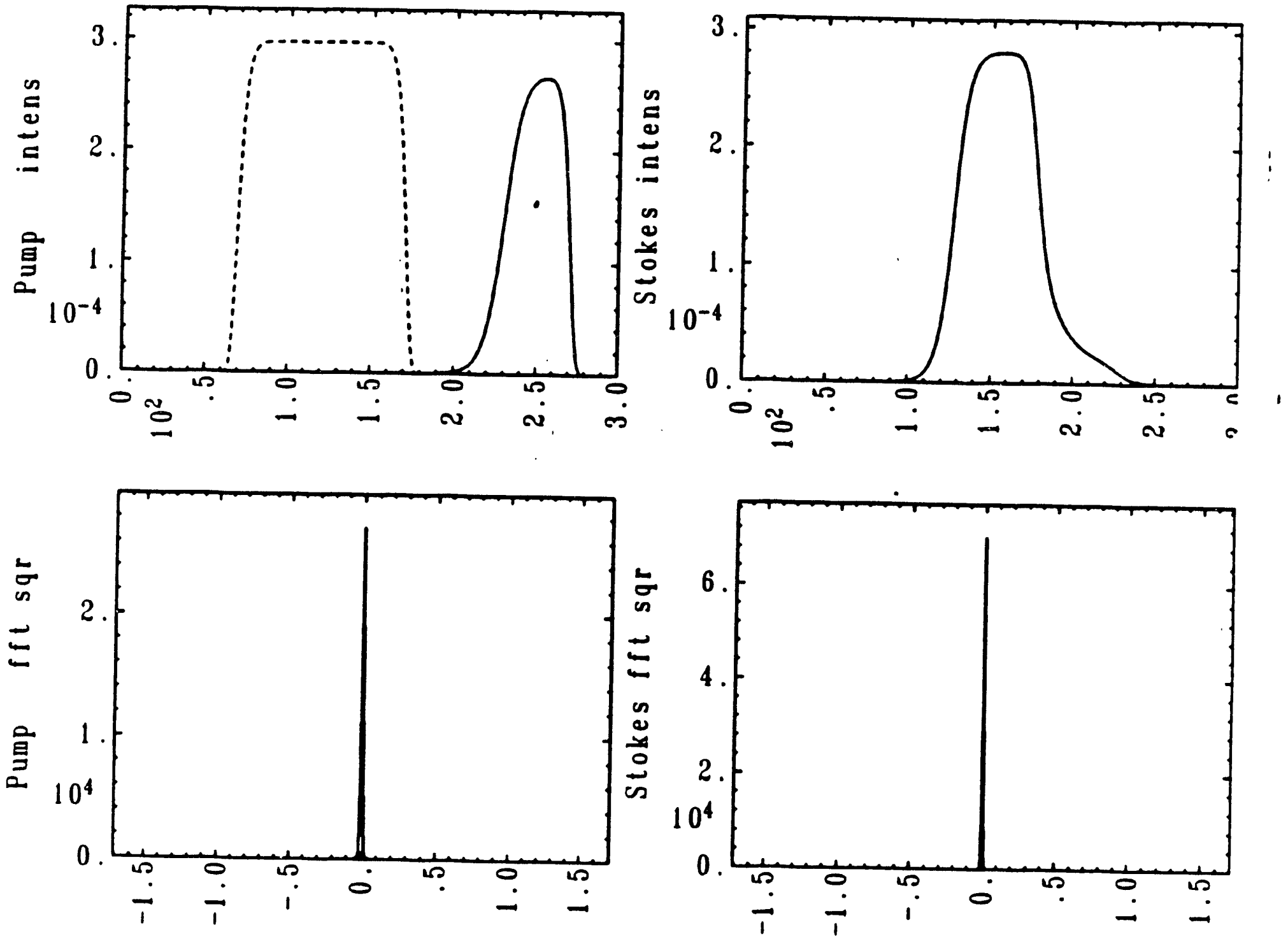

06-28-91 09:05:53h frase 15 byperg puap, tdisp $=5$ 
Dispersion + Warble

near square pulse, warble

Fig. 11a

short relax

const stokes seed

$T$ disp $=1$

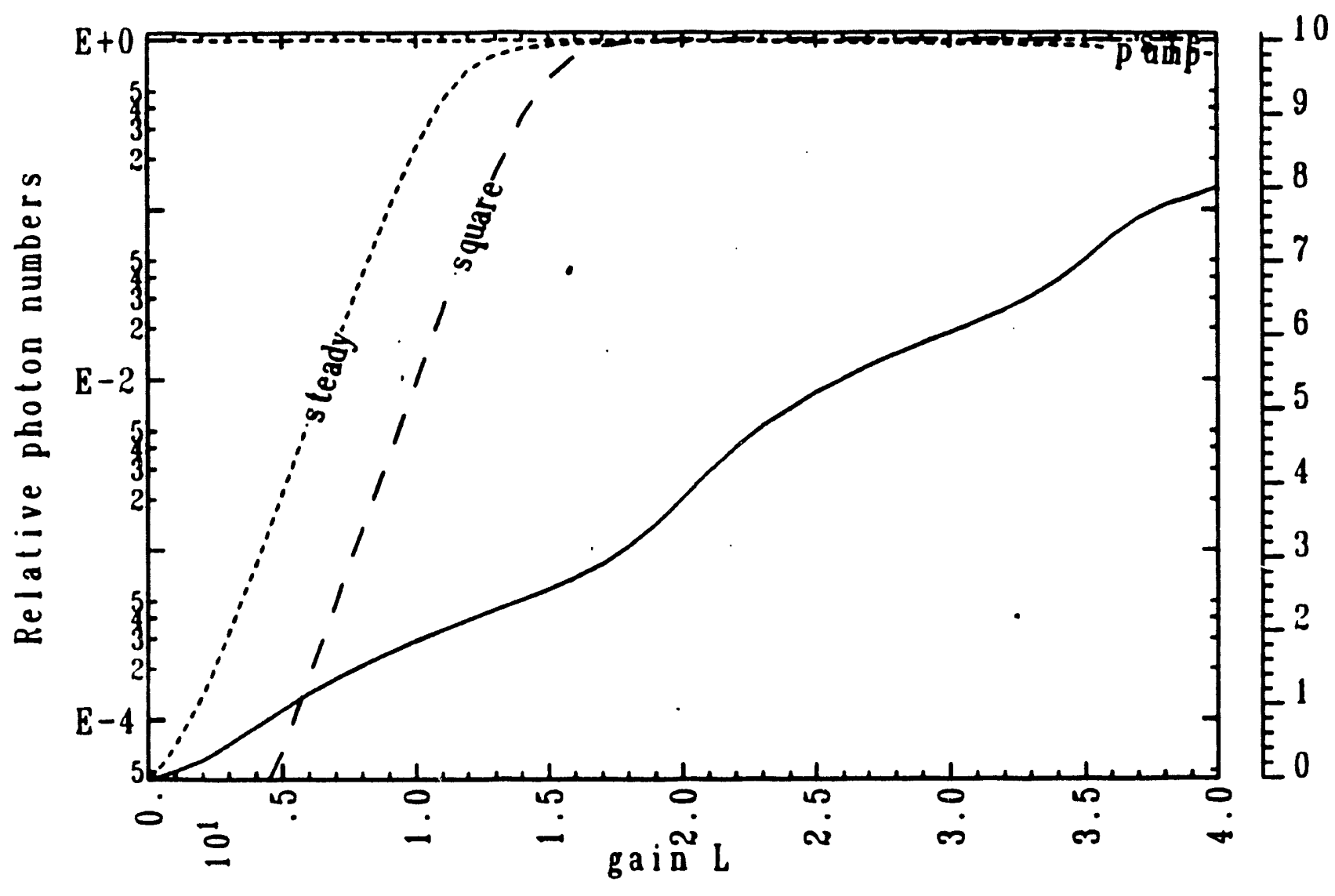



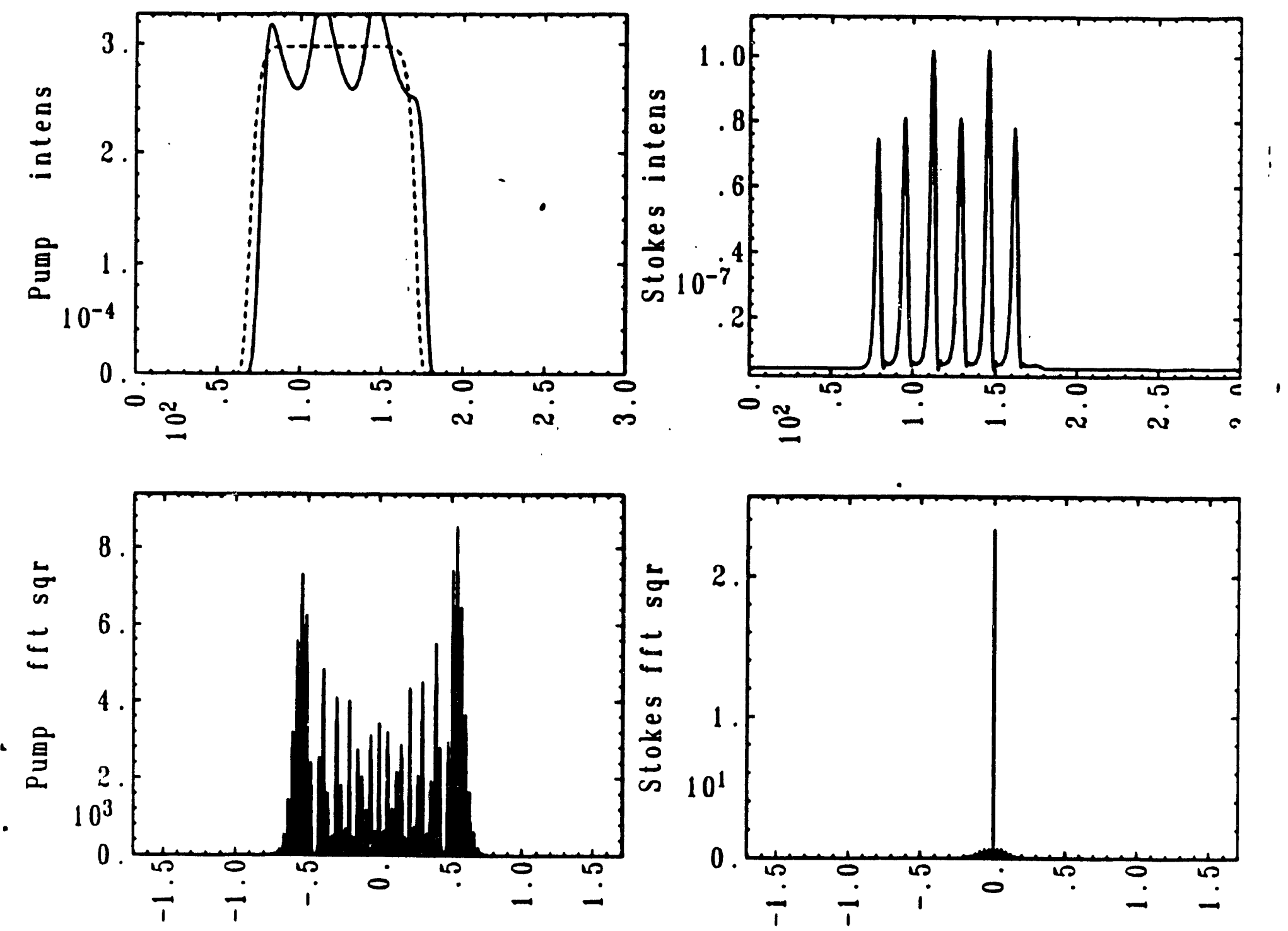

06-28-91 15:50:06 b frane 7 gauss/6 punp, warble, tdisp = 
near square pulse, warble

short relax

const stokes seed

$T$ disp $=1, \quad Z=40$

Pump

at $z=4.00 E+01$

Stokes
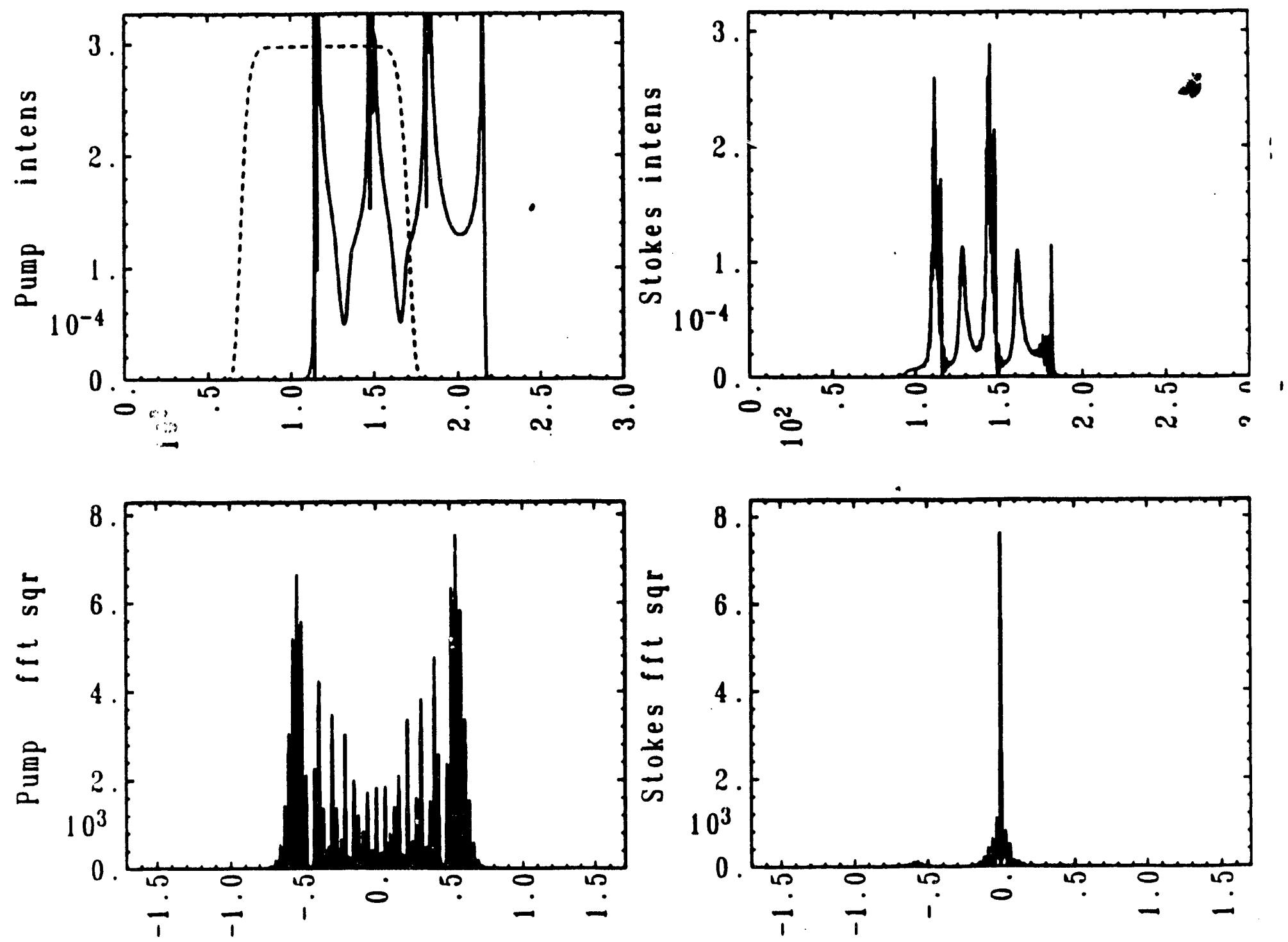

06-28-91 16:01:42b frame 19 gauss16 pump, warble, tdisp = 1 

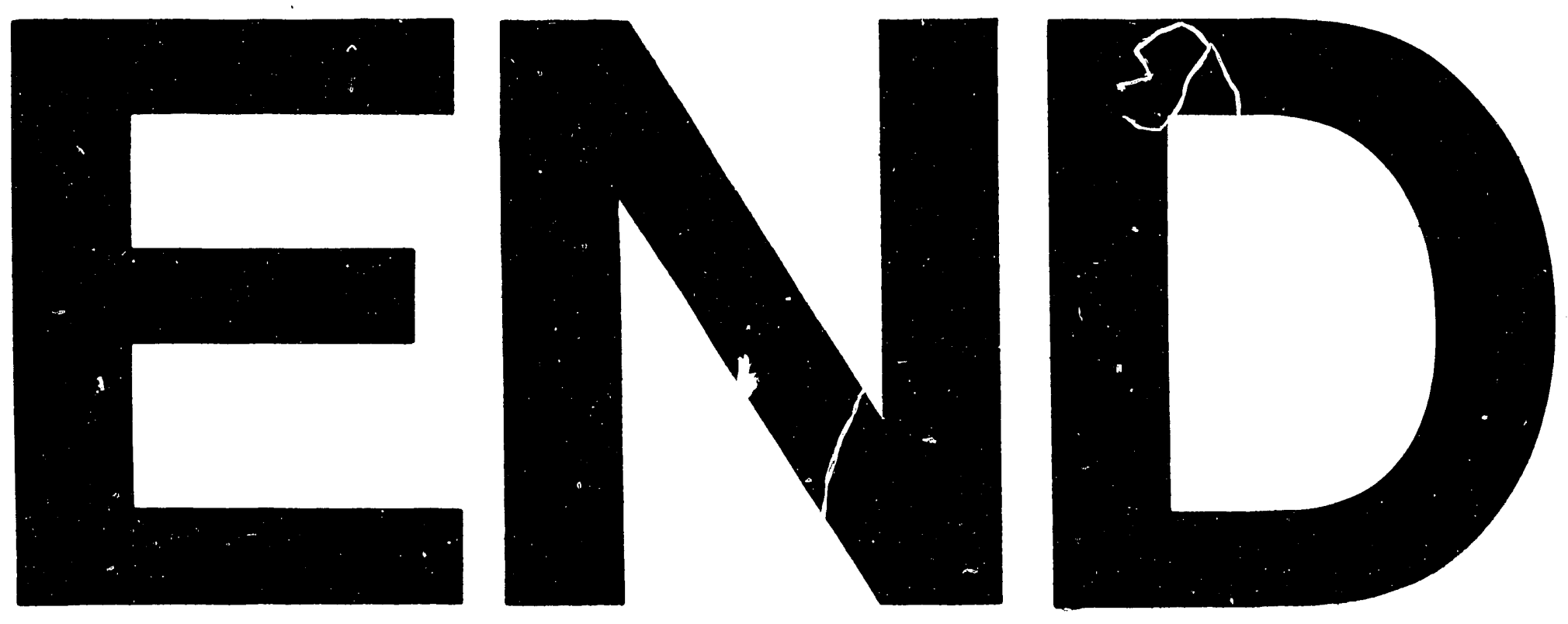

$\hat{i}$
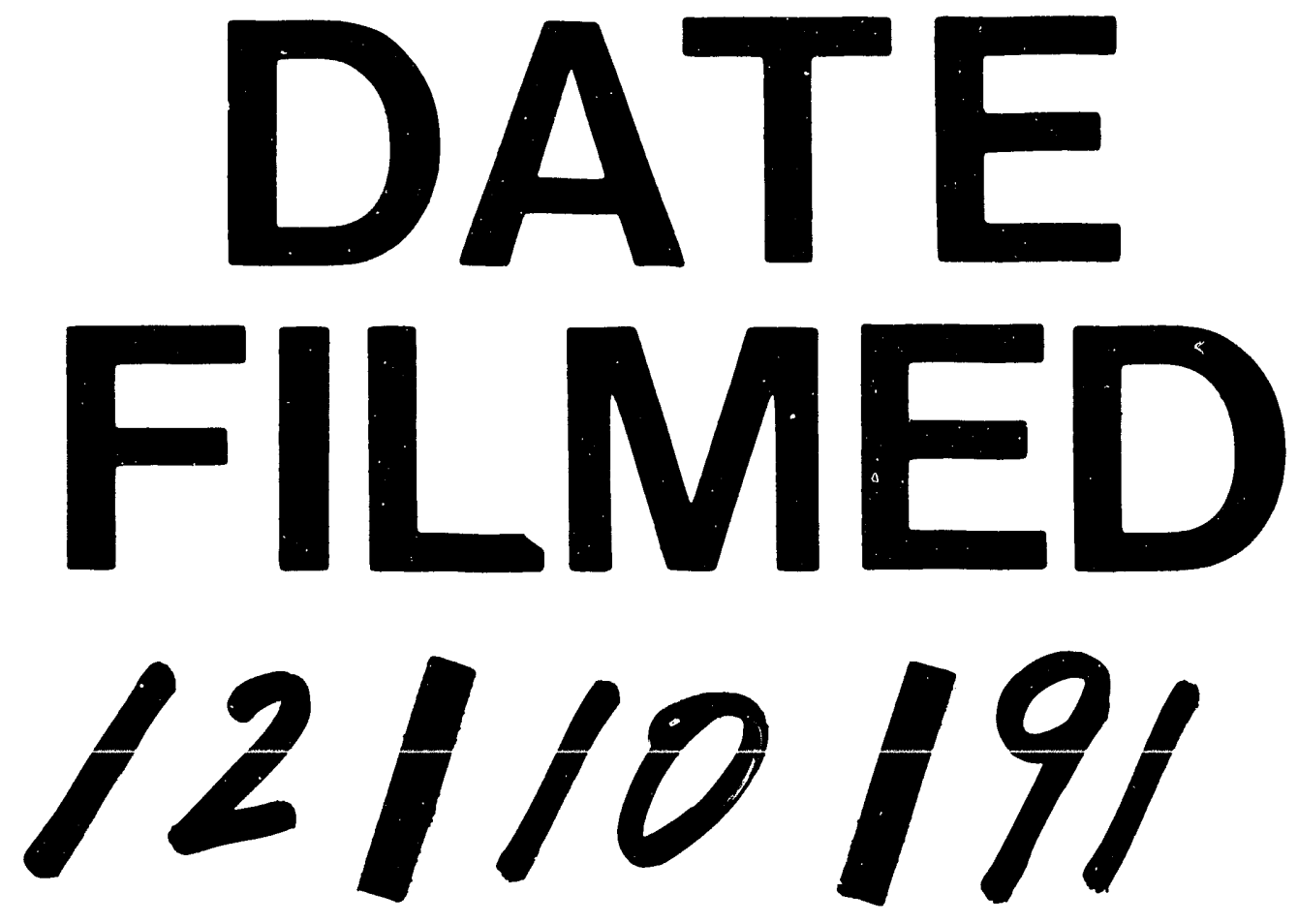

I 
